Artículo de opinión

\title{
Adaptación española de las guías europeas de 2016 sobre prevención de la enfermedad cardiovascular en la práctica clínica ${ }^{\text {tr }}$
}

\author{
Miguel Ángel Royo-Bordonada ${ }^{a, *}$, Pedro Armario ${ }^{b}$, José María Lobos Bejarano ${ }^{c}$, Juan Pedro-Botet ${ }^{\mathrm{d}}$, \\ Fernando Villar Álvarez ${ }^{\mathrm{d}}$, Roberto Elosua ${ }^{\mathrm{e}}$, Carlos Brotons Cuixart ${ }^{\mathrm{c}}$, Olga Cortés ${ }^{\mathrm{f}}$, Benilde Serrano ${ }^{\mathrm{g}}$, \\ Miguel Camafort Babkowski ${ }^{\mathrm{h}}$, Antonio Gil Núñez ${ }^{\mathrm{i}}$, Antonio Pérez ${ }^{j}$, Antonio Maiques ${ }^{\mathrm{c}}$, \\ Ana de Santiago Nocitok ${ }^{\mathrm{k}}$, Almudena de Castro ${ }^{1}$, Eduardo Alegría ${ }^{l}$, Ciro Baeza ${ }^{\mathrm{m}}$, María Herranz ${ }^{\mathrm{n}}$, \\ Susana Sans ${ }^{\circ}$ y Pilar Campos ${ }^{\mathrm{p}}$, en nombre del Comité Español Interdisciplinario para la Prevención \\ Cardiovascular (CEIPC) $)^{\diamond}$
}

\footnotetext{
ascuela Nacional de Sanidad, Instituto de Salud Carlos III, Madrid, España

b Sociedad Española de Hipertensión-Liga Española de la Lucha Contra la HTA

c Sociedad Española de Medicina de Familia y Comunitaria

d Sociedad Española de Arteriosclerosis

e Sociedad Española de Epidemiología

${ }^{\mathrm{f}}$ Asociación Española de Pediatría de Atención Primaria

$\mathrm{g}$ Sociedad Española de Medicina y Seguridad en el Trabajo

h Sociedad Española de Medicina Interna

i Sociedad Española de Neurología

j Sociedad Española de Diabetes

${ }^{\mathrm{k}}$ Sociedad Española de Médicos de Atención Primaria-Semergen

${ }^{1}$ Sociedad Española de Cardiología

m Sociedad Española de Angiología y Cirugía Vascular

${ }^{n}$ Federación de Asociaciones de Enfermería Comunitaria y Atención Primaria

- Sociedad Española de Salud Pública y Administración Sanitaria

p Ministerio de Sanidad, Servicios Sociales e Igualdad, Madrid, España
}

\section{INFORMACIÓN DEL ARTÍCULO}

\section{Historia del artículo:}

Recibido el 23 de noviembre de 2016

Aceptado el 23 de noviembre de 2016

On-line el 11 de marzo de 2017

\section{Palabras clave:}

Prevención y control

Enfermedades cardiovasculares

Guías de práctica clínica

Hipertensión arterial

Diabetes mellitus

Colesterol

Tabaco

Consumo de alcohol

Grasas trans

\section{R E S U M E N}

Las VI Guías Europeas de Prevención Cardiovascular recomiendan combinar las estrategias poblacional y de alto riesgo, con los cambios de estilo de vida como piedra angular de la prevención, y proponen la función SCORE para cuantificar el riesgo cardiovascular. Esta guía hace más hincapié en las intervenciones específicas de las enfermedades y las condiciones propias de las mujeres, las personas jóvenes y las minorías étnicas. No se recomienda el cribado de aterosclerosis subclínica con técnicas de imagen no invasivas. La guía establece cuatro niveles de riesgo (muy alto, alto, moderado y bajo), con objetivos terapéuticos de control lipídico según el riesgo. La diabetes mellitus confiere un riesgo alto, excepto en sujetos con diabetes tipo 2 con menos de 10 años de evolución, sin otros factores de riesgo ni complicaciones, o con diabetes tipo 1 de corta evolución sin complicaciones. La decisión de iniciar el tratamiento farmacológico de la hipertensión arterial dependerá del nivel de presión arterial y del riesgo cardiovascular, teniendo en cuenta la lesión de órganos diana. Siguen sin recomendarse los fármacos antiplaquetarios en prevención primaria por el riesgo de sangrado. La baja adherencia al tratamiento exige simplificar el régimen terapéutico e identificar y combatir sus causas. La guía destaca que los profesionales de la salud pueden ejercer un papel importante en la promoción de intervenciones poblacionales y propone medidas eficaces, tanto a nivel individual como poblacional, para promover una dieta saludable, la práctica de actividad física, el abandono del tabaquismo y la protección contra el abuso de alcohol.

(C) 2017 Publicado por Elsevier España, S.L.U. en nombre de SESPAS. Este es un artículo Open Access bajo la licencia CC BY-NC-ND (http://creativecommons.org/licenses/by-nc-nd/4.0/).

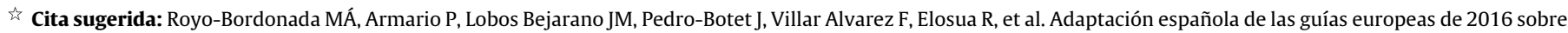
prevención de la enfermedad cardiovascular en la práctica clínica. Rev Esp Salud Pública. 2016;Vol.90: 24 de noviembre: e1-e24.

* Autor para correspondencia.

Correo electrónico: mroyo@isciii.es (M.Á. Royo-Bordonada).

URLs: http://www.ceipc.info (M.Á. Royo-Bordonada), http://www.isciii.es (M.Á. Royo-Bordonada).

$\checkmark$ Este documento también se ha publicado en las revistas órgano de expresión de las sociedades científicas del CEIPC en soporte papel o electrónico en línea y en la Revista Española de Salud Pública.
} 
Keywords:

Prevention and control

Cardiovascular diseases

Practice Guidelines [Publication Type]

Hypertension

Diabetes mellitus

Cholesterol

Tobacco

Alcohol drinking

Trans fatty acids

\section{Spanish adaptation of the 2016 European Guidelines on cardiovascular disease prevention in clinical practice}

\begin{abstract}
A B S T R A C T
The VI European Guidelines for Cardiovascular Prevention recommend combining population and highrisk strategies with lifestyle changes as a cornerstone of prevention, and propose the SCORE function to quantify cardiovascular risk. The guidelines highlight disease specific interventions, and conditions as women, young people and ethnic minorities. Screening for subclinical atherosclerosis with noninvasive imaging techniques is not recommended. The guidelines distinguish four risk levels (very high, high, moderate and low) with therapeutic objectives for lipid control according to risk. Diabetes mellitus confers a high risk, except for subjects with type 2 diabetes with less than $<10$ years of evolution, without other risk factors or complications, or type 1 diabetes of short evolution without complications. The decision to start pharmacological treatment of arterial hypertension will depend on the blood pressure level and the cardiovascular risk, taking into account the lesion of target organs. The guidelines don't recommend antiplatelet drugs in primary prevention because of the increased bleeding risk. The low adherence to the medication requires simplified therapeutic regimes and to identify and combat its causes. The guidelines highlight the responsibility of health professionals to take an active role in advocating evidence-based interventions at the population level, and propose effective interventions, at individual and population level, to promote a healthy diet, the practice of physical activity, the cessation of smoking and the protection against alcohol abuse.
\end{abstract}

(c) 2017 Published by Elsevier España, S.L.U. on behalf of SESPAS. This is an open access article under the CC BY-NC-ND license (http://creativecommons.org/licenses/by-nc-nd/4.0/).

\section{Introducción}

Las VI Guías Europeas de Prevención Cardiovascular representan el consenso basado en la evidencia del Sexto Grupo de Trabajo Conjunto Europeo, que incluye a 10 sociedades profesionales, para la prevención de la enfermedad cardiovascular (ECV) en la práctica clínica ${ }^{1}$. El grupo de trabajo ha usado el sistema de evaluación de la Sociedad Europea de Cardiología, basado en clases de recomendación y niveles de evidencia, aunque reconoce que puede ser menos adecuado para medir el impacto de intervenciones relacionadas con aspectos conductuales y, en particular, las estrategias poblacionales para modificar estilos de vida. Comparada con guías previas, ésta hace mayor hincapié en las intervenciones específicas de las enfermedades y las condiciones propias de las mujeres, las personas jóvenes y las minorías étnicas, y profundiza en el enfoque dirigido a la población, como ya hiciera el Comité Español Interdisciplinario de Prevención Cardiovascular (CEIPC) en la adaptación de la versión anterior de las guías ${ }^{2}$. La guía proporciona herramientas para los profesionales de la salud en la promoción de estrategias de base poblacional, integrarlas en contextos de prevención regional o nacional y trasladarlas a los servicios de salud locales. La traducción al castellano del texto completo de la guía, el anexo de intervenciones específicas dirigidas a enfermedades, con detalles útiles para la práctica clínica, y un artículo de la Sociedad Española de Cardiología con comentarios a la misma, se encuentran disponibles en http://www.revespcardiol.org/es/guia-esc-2016-sobreprevencion/articulo/90459676/.

En sus quince años de existencia, el CEIPC ha publicado la adaptación española de las guías europeas de 2003, 2008 y 2012 $2-4$, pasando de disponer de una decena de guías, con recomendaciones dispares, a un único documento consensuado y avalado por el Ministerio de Sanidad. En este documento presentamos la adaptación resumida y comentada de las Guías Europeas de Prevención Cardiovascular de $2016^{1}$, con el objetivo de promover su difusión e implementación para orientar la práctica clínica de los profesionales de la salud con un enfoque homogéneo. Siguiendo el esquema de la guía original, esta adaptación se estructura en torno a 4 cuestiones básicas: ¿qué es la prevención cardiovascular?, ¿quién debería beneficiarse de la prevención cardiovascular?, ¿cómo intervenir a nivel individual y poblacional? y ¿dónde intervenir a nivel individual y poblacional?

\section{¿Qué es la prevención cardiovascular?}

La prevención de la ECV se define como un conjunto de acciones coordinadas dirigidas a la población o a la persona con el fin de eliminar o minimizar el impacto de la ECV y las discapacidades asociadas $^{5}$. Es importante realizar un enfoque de por vida para controlar el riesgo cardiovascular (RCV). Esto implica que, además de mejorar el estilo de vida y reducir el nivel de los factores de riesgo de los pacientes con ECV establecida y aquellos en riesgo creciente de presentar ECV, se debe animar a las personas sanas de todas las edades a adoptar hábitos de vida saludables.

La ECV constituye la primera causa de muerte en la población española, originando en 2014 un total de 117.393 muertes, lo que supone el $30 \%$ de todas las defunciones ${ }^{6}$. La tendencia temporal en mortalidad ajustada por la edad en ECV se encuentra en descenso en los últimos 40 años (fig. 1). Por el contrario, las tasas de morbilidad hospitalaria por ECV casi se han triplicado en España durante ese periodo, aunque mostraron una disminución entre 2003 y 2012 (fig. 2) ${ }^{7}$. Más de la mitad de la reducción de la mortalidad cardiovascular se ha atribuido a cambios en el nivel de factores de riesgo en la población, especialmente la reducción de la colesterolemia, la presión arterial (PA) y el tabaquismo, una tendencia favorable contrarrestada parcialmente por el aumento de otros factores de riesgo, principalmente la obesidad y la diabetes mellitus tipo $2^{8}$.

La prevención es eficaz: si se practicara correctamente, la eliminación de conductas de riesgo podría evitar hasta un $80 \%$ de la ECV y un $40 \%$ de los cánceres ${ }^{9}$. Por tanto, resulta esencial mejorar la implementación de las medidas preventivas ${ }^{10}$. La mayor parte de episodios cardiovasculares se originan en personas con RCV moderado, sencillamente porque es el grupo de población más numeroso ${ }^{11}$. Por este motivo, las estrategias de prevención individuales para personas con riesgo alto o ECV establecida deben de complementarse con estrategias poblacionales que promuevan la adopción de estilos de vida saludables y la reducción de los factores de riesgo en la población ${ }^{12}$. Ambas estrategias son coste-efectivas, particularmente la legislación dirigida a disminuir el consumo de tabaco y el contenido de sal, azúcares y ácidos grasos de tipo trans en los alimentos, y los tratamientos para el control del colesterol y la presión arterial cuando se dirigen a personas con alto $\mathrm{RCV}^{13-19}$. Es importante señalar que el control poblacional de los principales factores de RCV es todavía muy bajo en España: 22,7\% para 


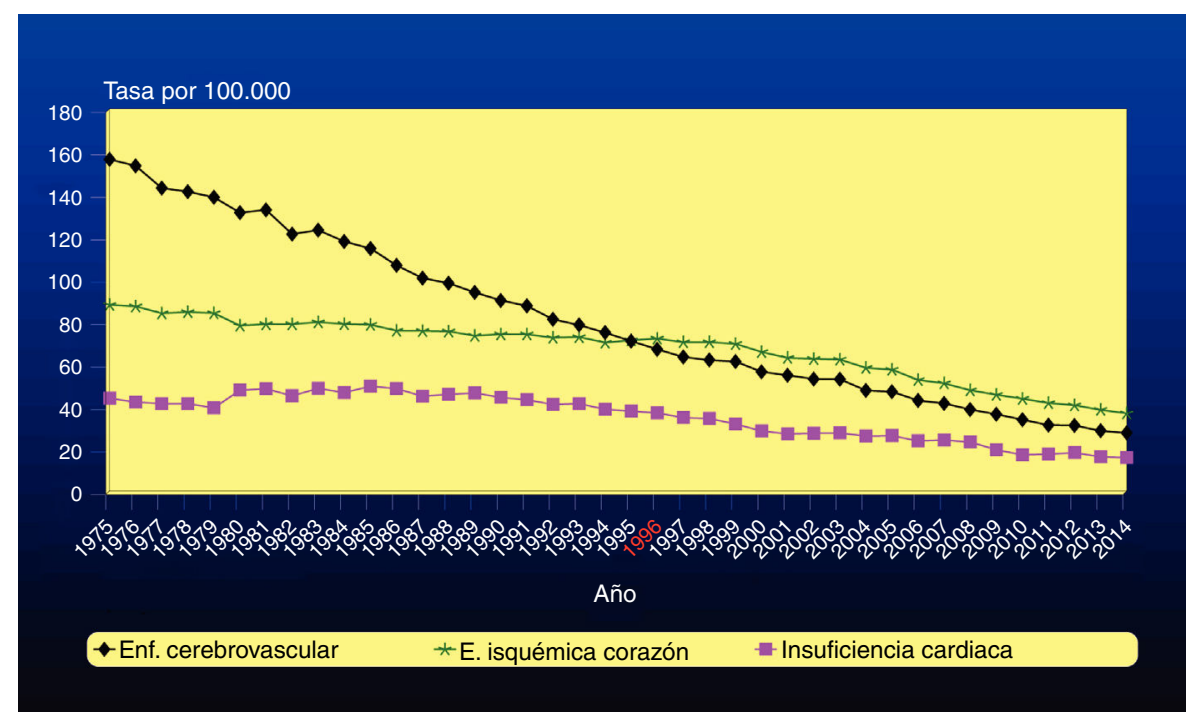

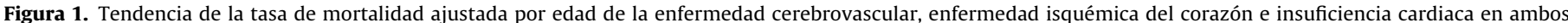
sexos. España, 1975-2014. Fuente: Actualización del Informe SEA 2007.

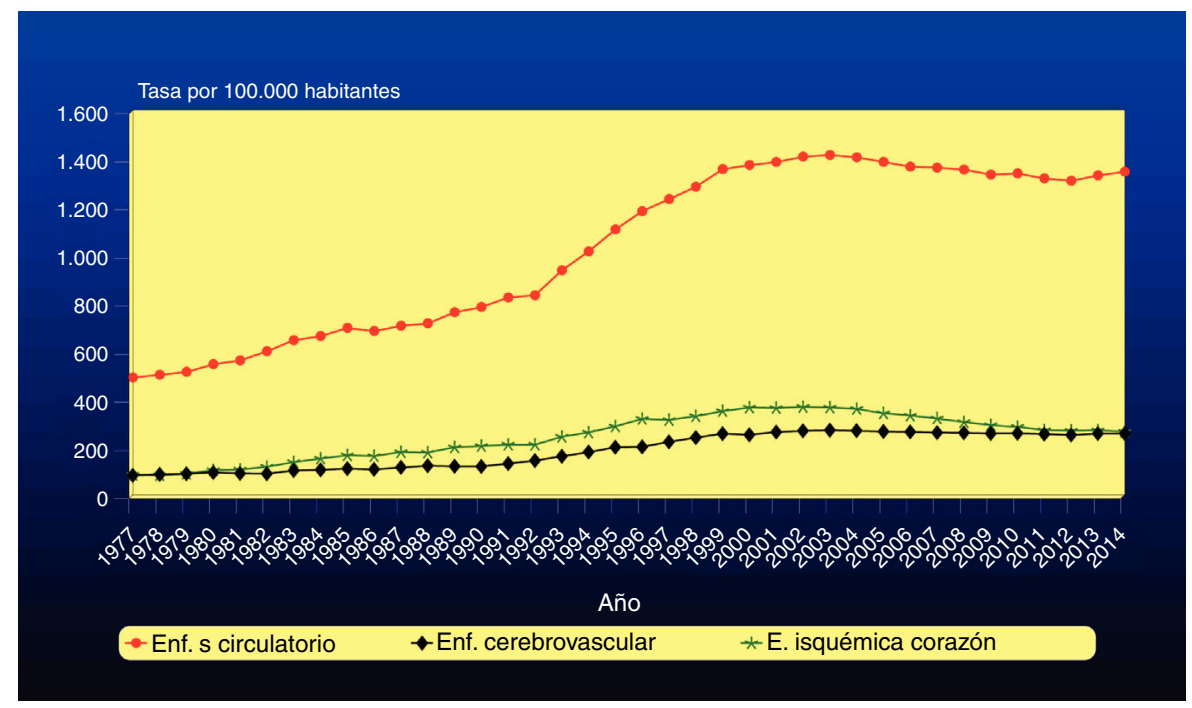

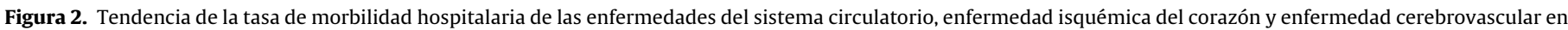
ambos sexos. España, 1977-2014. Fuente: Actualización del Informe SEA 2007.

la hipertensión arterial (HTA $)^{20}$ y $13,2 \%$ para el colesterol $^{21}$. Este hecho, junto con las diferencias territoriales en prevalencia y grado de control de factores de riesgo ${ }^{22}$, revelan el amplio margen existente aún para la prevención en nuestro entorno.

\section{¿Quién debería beneficiarse de la prevención cardiovascular?}

\section{Estrategias y estimación del riesgo}

El riesgo de presentar una ECV ateroesclerótica viene determinado por múltiples factores y sus interacciones. Por este motivo, desde la primera edición de las guías se estableció que el factor decisivo en la valoración de la intervención clínica fuera el riesgo de ECV: a mayor riesgo mayor la intensidad de las medidas preventivas a instaurar. En este sentido, meta-análisis recientes indican que el grupo de pacientes que más se beneficia de las estrategias preventivas a nivel de reducción absoluta de riesgo es el de riesgo más alto 23,24
La guía establece unos puntos de corte arbitrarios para definir RCV bajo, moderado, alto o muy alto, ya que el riesgo es un continuo, pero que ayudan en la toma de decisiones clínicas a nivel individual. Se recomienda estimar el RCV a personas que a priori tengan mayor riesgo (con antecedentes familiares de ECV prematura o dislipemias familiares, o pacientes que presenten un factor de riesgo importante - exposición al tabaco, diabetes, valores de presión arterial o lipídicos elevados -) y debería repetirse al menos cada 5 años. Podría considerarse también el cálculo del RCV en hombres de más de 40 años o mujeres de más de 50 años, o en la post-menopausia, que no presenten factores de riesgo, y no se recomienda en personas más jóvenes. Esta estrategia de cribado y estimación del RCV se puede implementar de forma oportunista (cuando el paciente contacta con el sistema sanitario independientemente del motivo) o de forma sistemática y planificada en subgrupos de población.

Las guías Europeas proponen la función SCORE para cuantificar el RCV, aunque también se incluyen otras funciones que hayan sido validadas a nivel local. En España existen diferentes funciones adaptadas y desarrolladas: SCORE calibrado para la población española ${ }^{25}$, REGICOR $^{11}$, FRESCO $^{26}$ y ERICE $^{27}$, aunque únicamente 


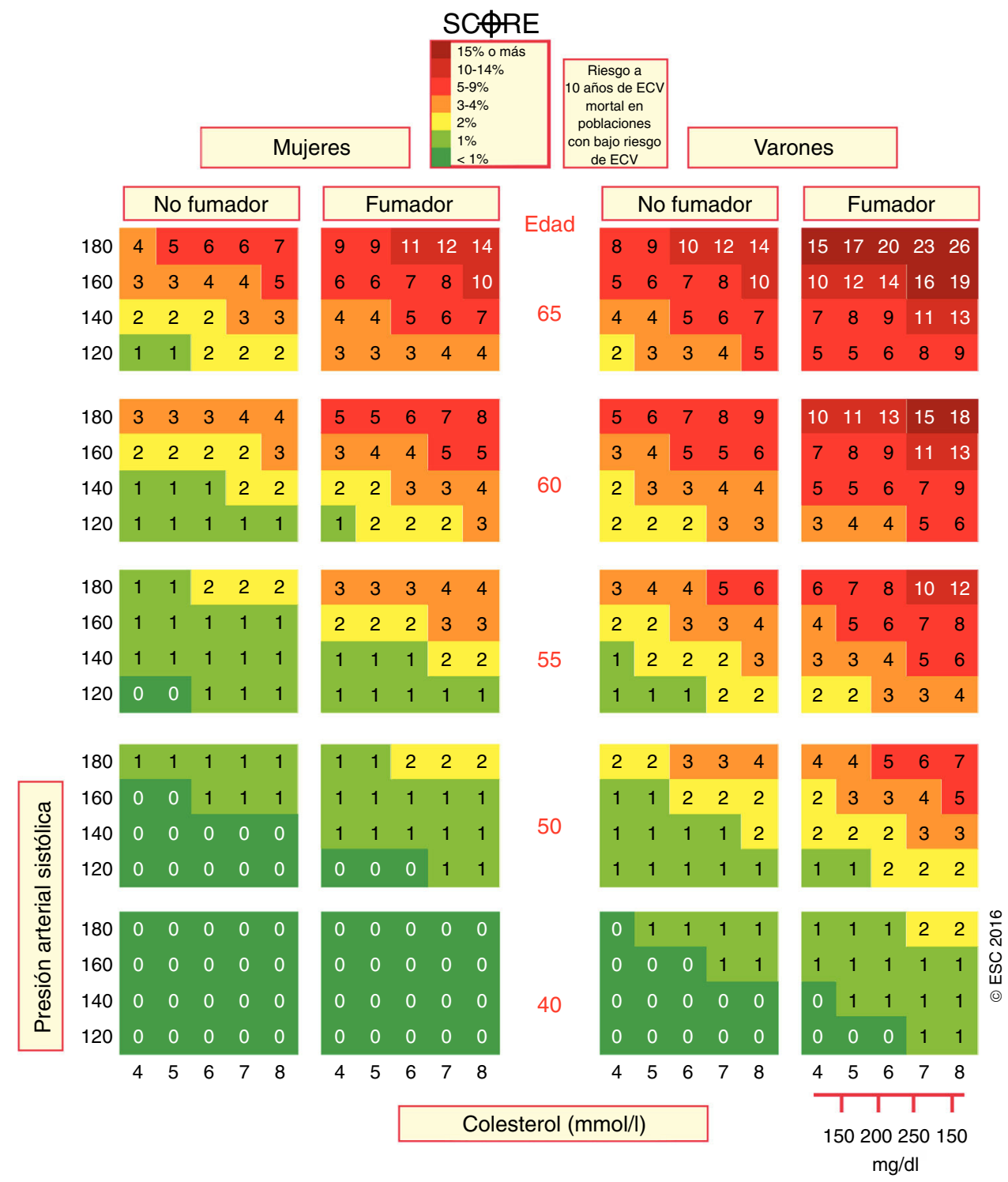

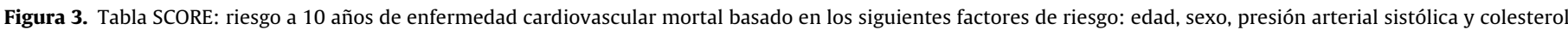
total. ECV: enfermedad cardiovascular; SCORE: Systematic Coronary Risk Estimation.

tenemos datos de la validez de dos de ellas ${ }^{11,26,28}$. En la mayoría de CCAA se utiliza SCORE, pero en otras se utiliza la función REGICOR e incluso Framingham original ${ }^{29}$. Sin embargo, más allá de la función que se utilice para su cálculo, lo más importante es estimar el RCV como estrategia de prevención cardiovascular en la práctica clínica y aplicar las recomendaciones de las guías sobre la base del juicio clínico y las preferencias del paciente. Para calcular el riesgo de muerte CV a 10 años de una persona, hay que buscar la tabla (fig. 3) de su mismo sexo, si consume tabaco y su edad (la más próxima). Dentro de la tabla, hay que buscar la celda que esté más próxima a la PA y el colesterol total de esa persona. El cálculo de riesgo tendrá que ajustarse al alza a medida que la persona se acerca a la siguiente categoría de edad.

Los sistemas o funciones de estimación del riesgo son instrumentos relativamente sencillos que deberían implementarse en la historia clínica electrónica, pero tienen una serie de características, utilidades y limitaciones que deben ser consideradas cuando se utilizan en la práctica clínica diaria:

a) El RCV es una variable continua que nos informa sobre el riesgo absoluto, a mayor riesgo estimado mayor incidencia de episodios cardiovasculares, por lo que no existe un umbral claro a partir del cual se pueda decidir iniciar tratamiento farmacológico o una intervención individualizada sobre el estilo de vida. Las guías definen cuatro categorías de riesgo: bajo (SCORE <1\%), moderado (SCORE $\geq 1 \% \mathrm{y}<5 \%$ ), alto (SCORE $\geq 5 \% \mathrm{y}<10 \%$ ) y muy alto (SCORE $\geq 10 \%$ ).

b) El cálculo del riesgo en personas jóvenes. En personas jóvenes aunque presenten muchos factores de riesgo, el RCV suele ser bajo o moderado. Para intentar comunicar el riesgo en este grupo de personas se recomienda calcular el riesgo relativo, la edad de riesgo vascular y el riesgo a lo largo de la vida. El riesgo relativo informa sobre cuantas veces más riesgo tiene el paciente respecto de una persona de su misma edad y sexo pero sin factores de riesgo. La edad de riesgo vascular o edad vascular de un paciente es la edad a la que una persona sin factores de riesgo alcanzaría el riesgo que presenta actualmente ese paciente ${ }^{30,31}$. A modo de ejemplo, un varón de 50 años fumador y con $250 \mathrm{mg} / \mathrm{dl}$ de colesterol tiene una edad vascular de 65 años. El riesgo a lo largo de la vida también puede ser una estrategia válida en este subgrupo de la población, pero en España no disponemos todavía de funciones que permitan su cálculo. Estos 
indicadores de riesgo pueden ser muy útiles como elementos para la comunicación médico-paciente y quizás también servir en la toma de decisiones en casos dudosos y de acuerdo con el paciente.

c) El cálculo del riesgo en personas mayores. Hay que tener en cuenta que la edad es el principal determinante del RCV para evitar el sobretratamiento con fármacos en las personas ancianas, sobre todo dada la escasa evidencia de las estrategias preventivas en este grupo de edad. Recientemente, los investigadores de la SCORE han publicado unas tablas específicas para pacientes mayores de 65 años $^{32}$. El uso de estas tablas en un grupo de 974 pacientes españoles de 65 a 69 años identificó menos pacientes de alto y muy alto riesgo que con las tablas originales, lo que implicaría la aplicación de tratamiento farmacológico en un número potencialmente menor de pacientes ${ }^{33}$.

d) En el cálculo del RCV se debe prestar atención a otros factores no incorporados en la función de riesgo, pero que se pueden obtener fácilmente en la práctica clínica habitual, sobre todo en individuos en los límites de una categoría de riesgo, que pueden pasar a un grupo de riesgo más elevado y requerir estrategias preventivas más intensas. Entre estos factores modificadores del riesgo se incluyen los antecedentes familiares de ECV prematura, la presencia de obesidad general o central y factores psicosociales como la posición socioeconómica, el aislamiento social y la falta de apoyo social. Aunque uno de los problemas en la práctica clínica diaria con los factores psicosociales es su evaluación con métodos estandarizados, la guía propone una serie de preguntas sencillas que pueden incluirse en la rutina diaria. El cHDL tiene un efecto pequeño, aunque útil, en la redefinición del cálculo de riesgo, y la versión electrónica del sistema SCORE, el HeartScore (http://www.HeartScore.org), se ha modificado para tener en cuenta las mediciones de cHDL.

\section{Otros marcadores de riesgo}

Una de las limitaciones de las funciones de riesgo es su baja sensibilidad $^{11}$. Por este motivo en los últimos años se están evaluando nuevos marcadores que mejoren la capacidad predictiva de las funciones clásicas ${ }^{34}$. La guía contiene una serie de recomendaciones sobre la utilización de algunos de estos nuevos marcadores: genéticos, plasmáticos o urinarios, de daño vascular preclínico y otras entidades clínicas que influyen en el RCV.

\section{Biomarcadores genéticos}

En los últimos años se ha avanzado en el conocimiento de la arquitectura genética de la ECV (genes y variantes genéticas importantes). Las variantes genéticas identificadas presentan una magnitud de asociación con la ECV pequeña (OR: $1,1-1,25)$ y para evaluar su capacidad predictiva se necesitan más estudios que confirmen estos resultados, por lo que no se recomienda utilizar pruebas genéticas en la evaluación del RCV.

\section{Biomarcadores plasmáticos y urinarios}

Se clasifican en inflamatorios (e.g. proteína $C$ reactiva), trombóticos (e.g. fibrinógeno), relacionados con la glucosa y los lípidos (e.g. apolipoproteínas) y específicos de órgano (e.g. renales o cardiacos). Los datos del valor añadido en la capacidad predictiva de estos biomarcadores varían mucho entre estudios y existe un posible sesgo de publicación de resultados positivos ${ }^{35}$. Su uso rutinario no está recomendado por las guías, y si se utilizaran se tendría que tener en cuenta que el ajuste en la estimación del riesgo absoluto debería de ser pequeño y la reclasificación sólo debería afectar a individuos con riesgo moderado y en los límites del punto de corte de la categoría superior o inferior.

\section{Medida de la enfermedad vascular preclínica}

El cribado de aterosclerosis subclínica con técnicas de imagen no invasivas no está recomendado en la práctica clínica. No obstante, los métodos de imagen pueden considerarse modificadores del riesgo en personas con RCV próximo al umbral de decisión. La medición del calcio coronario basándose en el uso de la puntuación de Agatston es un marcador independiente de riesgo coronario y de su pronóstico, principalmente porque su determinación muestra un valor predictivo negativo muy elevado ${ }^{36}$. No obstante, la radiación que supone, la falta de precisión para el análisis funcional de las lesiones y la relación coste-beneficio, impiden su recomendación. Los estudios sobre la capacidad de los ultrasonidos (ecografía carotídea) para predecir el RCV mediante medición del grosor íntima-media muestran resultados inconsistentes ${ }^{37,38}$, por lo que no se recomienda su uso. Los pacientes con placas estenóticas ecolucentes tienen un RCV más elevado que individuos con otro tipo de placas. El análisis de la placa carotídea puede considerarse como un modificador del RCV en algunos casos. La rigidez arterial medida en función de la velocidad de la onda de pulso en la aorta puede relacionarse con el grado de lesión en la pared arterial en pacientes hipertensos, pero su uso como predictor del RCV no está recomendado. El ITB tiene una relación inversa con el RCV, pero existe controversia sobre su capacidad para reclasificar a los pacientes en categorías de riesgo diferentes ${ }^{39}$. Finalmente, la ecocardiografía no está recomendada para mejorar la predicción del RCV por las dificultades de realización y la falta de evidencia de su capacidad para reclasificar pacientes.

\section{Condiciones clínicas que influyen en el RCV}

La enfermedad renal crónica se asocia con un mayor RCV, independientemente de los factores de riesgo convencionales. La HTA, la dislipemia y la diabetes son comunes en pacientes con esa condición ${ }^{40}$. Hay asociación entre las infecciones respiratorias agudas, especialmente las que se producen en los momentos álgidos de la circulación del virus de la gripe, y el infarto agudo de miocardio, por lo que se puede considerar la vacunación antigripal anual en pacientes con ECV establecida ${ }^{41}$. Los estudios han relacionado la enfermedad periodontal con la aterosclerosis y la ECV ${ }^{42}$. Los pacientes que sobreviven al cáncer después del tratamiento con quimioterapia o radioterapia tienen un mayor RCV. El aumento de la incidencia de ECV se correlaciona con la combinación de los tratamientos y la dosis administrada. Por lo tanto, se debe considerar la optimización del RCV en los pacientes tratados de cáncer y la cardioprotección en pacientes de alto riesgo que reciben quimioterapia de tipo I para la prevención de la disfunción del ventrículo izquierdo ${ }^{43}$. La artritis reumatoide aumenta el RCV un $50 \%$, por lo que se debe considerar la multiplicación del RCV por $1,5^{44}$. Otras enfermedades inmunológicas, como la espondilitis anquilosante o la psoriasis precoz grave, también se pueden considerar multiplicadoras del RCV en el mismo grado, dependiendo de su actividad/gravedad. Hay evidencia de una relación positiva entre el síndrome de apnea obstructiva del sueño y la HTA, la enfermedad coronaria, la fibrilación auricular, el ictus y la insuficiencia cardiaca, pero se necesitan más estudios para determinar si su cribado reduce la $\mathrm{ECV}^{45}$. Por último, la disfunción eréctil se asocia con futuros episodios cardiovasculares en hombres con y sin ECV establecida, por lo que se debe considerar la evaluación del RCV en varones afectados, pero el beneficio del cribado de rutina y la herramienta más eficaz para evaluarla no están claros $^{46}$.

\section{Grupos relevantes}

\section{Sujetos de edad inferior a 50 años}

Algunos sujetos presentan un RCV alto, debiendo ofrecérseles consejos de cambios en el estilo de vida. Además en determinados individuos apreciamos factores de riesgo aisladamente elevados 
(como niveles de colesterol $>300 \mathrm{mg} / \mathrm{dl}$ o $\mathrm{PA} \geq 180 / 110 \mathrm{~mm} \mathrm{Hg}$ ), que imponen una intervención. En este grupo debemos necesariamente identificar aquellos con historia familiar de ECV prematura, y aquellos con posible dislipemia familiar ${ }^{47}$. Cuestiones pendientes son cuándo comenzar la estimación del RCV y cuándo y cómo detectar poblaciones con dislipemia familiar.

\section{Ancianos}

Aunque la edad per se es un factor de riesgo, los tratamientos aplazan la morbilidad y la mortalidad. Por ello se recomienda una discusión detallada con los pacientes en cuanto a años de vida y calidad de vida potencialmente ganada con las intervenciones, así como con respecto a la polifarmacia, los efectos adversos y las incertidumbres de beneficio. Se recomiendan objetivos de tratamiento menos intensivos en HTA y diabetes, debiendo tenerse en cuenta en el balance beneficio/efectos adversos del tratamiento con estatinas en dislipemia ${ }^{48}$.

\section{Mujeres}

Debe tenerse en cuenta que la preeclampsia y la HTA relacionada con el embarazo están asociados a un mayor riesgo de ECV en el futuro ${ }^{49}$. Asimismo, el síndrome de ovario poliquístico confiere un mayor riesgo de desarrollo de diabetes ${ }^{50}$. Aspectos sin resolver son si dichas condiciones propias de la mujer se comportan como factores de riesgo independientes de los clásicos. Además, desconocemos si la presencia de dichas situaciones puede mejorar la clasificación de riesgo en mujeres.

\section{Minorías étnicas}

Debemos tener en cuenta que el RCV varía considerablemente entre los grupos de inmigrantes: los procedentes del sur de Asia y los subsaharianos tienen un riesgo más alto, mientras que chinos y sudamericanos tienen un menor riesgo. Asimismo, los procedentes del sur de Asia se caracterizan por una alta prevalencia e inadecuado manejo de la diabetes. Sobre todo debemos también tener en cuenta que las ecuaciones de estimación de riesgo actuales no proporcionan estimaciones adecuadas de RCV en las minorías étnicas $^{51}$. Por ello, necesitamos estudios enfocados en la prevalencia de factores de RCV en dichas minorías, así como una validación del SCORE, que permitan definir que valores definen situaciones de alto riesgo en diferentes grupos étnicos.

\section{¿Cómo intervenir a nivel individual?}

\section{Cambio de comportamiento}

Los métodos de intervención cognitivo-conductual son eficaces para motivar el cambio e inducir a la adopción de un estilo de vida saludable ${ }^{52}$. En concreto, la intervención para el fomento de la salud en equipos multidisciplinares (enfermeros, dietistas, psicólogos, ...) es una medida eficaz para mejorar los hábitos higiénico dietéticos y el perfil cardiovascular ${ }^{53}$. En los pacientes con RCV muy elevado se recomienda una intervención que combine los recursos médicos, educación sobre estilos de vida, actividad física, manejo del estrés y factores de riesgo psicosociales. Sin embargo, todavía hay escasa evidencia que determine qué intervenciones son las más eficaces para modificar los estilos de vida en grupos específicos (por ejemplo según la edad, el sexo y el nivel socioeconómico).

\section{Factores psicosociales}

En pacientes con ECV establecida y síntomas psicosociales (estrés, aislamiento social y emociones negativas) se recomienda realizar intervenciones conductuales para mejorar su nivel de salud psicosocial, integrando educación para la salud, ejercicio físico y terapia psicológica ${ }^{54}$. En presencia de síntomas clínicamente significativos de depresión, ansiedad o agresividad, se debe remitir a los pacientes para recibir psicoterapia, tratamiento farmacológico o cuidados a un equipo multidisciplinar ${ }^{55}$. El tratamiento de factores de riesgo psicosocial se debe de valorar con la intención de prevenir la enfermedad coronaria, cuando sean por sí mismos una alteración diagnosticable (por ejemplo, una depresión) o cuando empeoren los factores de riesgo clásicos ${ }^{56}$. No se disponen de resultados concluyentes sobre la evidencia de que el tratamiento de la depresión y la ansiedad por sí solo evite la ECV.

\section{Sedentarismo y actividad física}

Las personas de cualquier edad y condición que practican actividad física regularmente tienen menor riesgo de complicaciones y de mortalidad cardiovascular. Este efecto es debido a la influencia favorable del ejercicio habitual sobre muchos factores de RCV (HTA, lipoproteínas aterógenas, peso y diabetes) y a la mejora de la capacidad física y la salud mental ${ }^{57,58}$. El estilo de vida sedentario, en consecuencia, está considerado como uno de los factores de riesgo principales de ECV.

En las guías europeas se recomienda la práctica de ejercicio aerobio a todos los adultos sanos de cualquier edad. Llama la atención que esta recomendación no se extienda también a niños y adolescentes y que no se haga mención a este colectivo en el apartado de grupos relevantes, cuando precisamente la prevención en este momento vital es igual o más importante que en la edad adulta y mucho más que en la ancianidad, a la que se dedica un amplio apartado específico. El mínimo recomendado es de 150 minutos/semana ( 30 minutos diarios cinco días a la semana) de ejercicio de intensidad moderada o 75 minutos/semana de ejercicio vigoroso. Idealmente esta cantidad debería duplicarse paulatinamente para obtener el máximo beneficio preventivo. Caben lógicamente otras modalidades de entrenamiento o combinaciones de ejercicios aerobios e isométricos, con vigilancia regular. Está por comprobar la utilidad de los sistemas de control del ejercicio mediante supervisión periódica o con métodos a distancia (monitores portables, aplicaciones para teléfonos, etc.), pero no es descabellado pensar que la tienen.

El riesgo de complicaciones cardiovasculares durante el ejercicio es extremadamente bajo (5-17 fallecimientos/millón/año, según la intensidad del mismo) en adultos aparentemente sanos ${ }^{59}$. Por ello, en los pacientes de bajo riesgo no sería necesario un estudio cardiológico previo; sí sería pertinente estudio cardiológico y ergométrico previo en personas sedentarias que pretenden iniciar un plan de entrenamiento exigente.

\section{Tabaquismo}

Se recomienda no fumar tabaco ni otros productos derivados de hierbas ya que su consumo aumenta la prevalencia de $\mathrm{ECV}^{60}$. Asimismo, se recomienda evitar la exposición al humo del tabaco (fumador pasivo) ya que eleva de forma significativa el riesgo de $\mathrm{ECV}^{61}$. En fumadores, el abandono del tabaco es la medida más eficiente para prevenir la ECV. Por lo tanto, se recomienda identificar a los fumadores y aconsejar de modo sistemático el abandono del tabaco, ofrecer ayuda que incluya seguimiento, terapias de sustitución de nicotina, varenciclina y bupropion, tanto en tratamiento con un sólo fármaco como en combinación ${ }^{62}$. Hay evidencias de una mayor eficacia de los tratamientos farmacológicos combinados, excepto para las terapias de reemplazo de nicotina más vareniclina. Las medidas terapéuticas más eficaces se basan en la combinación de breves intervenciones y el uso de fármacos durante el tiempo inicial de abandono del hábito y el seguimiento. 
Los cigarrillos electrónicos pueden ser útiles en el inicio del abandono del hábito, pero su uso debería de estar sujeto a las mismas restricciones de comercialización que los cigarrillos ${ }^{63}$. Sin embargo, no existen datos fiables que la acupuntura, la acupresión, la terapia con láser, la hipnoterapia o la electroestimulación sean efectivos para dejar de fumar.

\section{Nutrición}

La piedra angular de la prevención cardiovascular es una dieta saludable, que comienza por limitar la ingesta energética para mantener un peso saludable.

\section{Nutrientes}

La composición de la grasa de la dieta es más importante que la cantidad total ingerida, destacando la reducción del RCV asociada al consumo de ácidos grasos poliinsaturados y el efecto pernicioso de los ácidos grasos trans de origen industrial, cuyo consumo se recomienda evitar. En España, su ingesta media está entre 2 y 2,5 g/día ( $1 \%$ de la ingesta calórica total) ${ }^{64}$, una cantidad asociada con un incremento del riesgo de enfermedad coronaria superior al $10 \% 65$. Mientras que la ingesta de fibra (30-45 g/día) reduce el riesgo de diabetes y ECV, la de azúcares añadidos aumenta la mortalidad cardiovascular $^{66}$. Reducir la ingesta de sal es eficaz para bajar la presión arterial en pacientes hipertensos y normotensos, por lo que se recomienda consumir menos de $5 \mathrm{~g}$ /día. No obstante, estudios recientes sugieren que el efecto protector de la reducción de la ingesta de sal en la enfermedad y mortalidad cardiovasculares podría limitarse a los pacientes hipertensos con alto consumo de sodio $^{67}$. La presión arterial también puede reducirse aumentando el consumo de potasio. Los ensayos clínicos no han encontrado efectos beneficios ni de los suplementos de vitaminas (A, B, C, D o E) ni del aceite de pescado en prevención cardiovascular.

\section{Alimentos}

La ingesta diaria de frutas y verduras ( $\geq 5$ al día) y de frutos secos ( $30 \mathrm{~g} /$ día) disminuye hasta un $30 \%$ el RCV, y el consumo de pescado al menos una vez por semana reduce un $16 \%$ el riesgo de enfermedad coronaria. Los alimentos funcionales con fitoesteroles $(2 \mathrm{~g} /$ día $)$ reducen hasta un $10 \%$ los niveles de colesterol LDL, aunque no hay estudios clínicos con episodios cardiovasculares. El consumo regular de bebidas azucaradas aumenta el riesgo de obesidad y enfermedad coronaria (20\% mayor con una bebida diaria) ${ }^{68}$, y el consumo excesivo de alcohol se asocia con un RCV alto, por lo que se desaconseja el consumo de bebidas azucaradas y alcohólicas.

\section{Patrones alimentarios}

El riesgo de ECV y su mortalidad asociada son más bajos cuanto mayor es la adherencia a la dieta mediterránea (abundancia de productos de origen vegetal frescos o mínimamente procesados -frutas, verduras, cereales, legumbres, frutos secos-, escasez de productos ricos en azúcares y carnes rojas, aceite de oliva como principal fuente de grasa, ingesta de queso, yogurt, pollo y pescado en cantidades moderadas, y consumo moderado de vino en las comidas), un patrón alimentario que puede servir como modelo de referencia para la prevención cardiovascular ${ }^{69}$.

\section{Peso corporal}

A diferencia de la evolución favorable de los principales factores de RCV, como colesterol, presión arterial y tabaquismo, el índice de masa corporal (IMC) ha mantenido un continuado ascenso en todos los países durante las últimas décadas. Dado que el RCV tiene una relación positiva continua con el IMC y otras medidas de grasa corporal, se recomienda que los sujetos con un peso saludable lo mantengan, y aquellos con sobrepeso u obesidad, alcancen un peso saludable o aspiren a una disminución ponderal ${ }^{70,71}$.

En relación con la grasa abdominal, se debe recomendar no ganar peso cuando el perímetro de cintura $\geq 94 \mathrm{~cm}$ en hombres y $80 \mathrm{~cm}$ en mujeres, y la reducción de peso si perímetro de cintura es $\geq$ a $102 \mathrm{~cm}$ en hombres y $88 \mathrm{~cm}$ en mujeres. El IMC y el perímetro de cintura se asocian de forma continua y con la misma fuerza con ECVy diabetes, por lo que la medición del IMC es suficiente generalmente en la práctica clínica habitual ${ }^{72}$.

A pesar de que la dieta, el ejercicio y las modificaciones de la conducta son los pilares del tratamiento del sobrepeso y la obesidad, estas medidas fracasan frecuentemente en el tratamiento a largo plazo. En España, aunque en la historia clínica electrónica de las distintas CCAA se recogen datos antropométricos (peso, talla, IMC, perímetro de cintura e índice cadera/cintura), siguen sin incluirse indicadores del grado de control de la obesidad, dieta, ejercicio físico y cambios de conducta en los contratos programa, que permitan una correcta valoración y seguimiento de la obesidad, y establecer posibles medidas correctoras ${ }^{29}$.

\section{Lípidos}

La presenta guía presenta sutiles cambios con respecto a la del $2012^{73}$. Cabe destacar que se recomienda que el RCV guie la intensidad de la estrategia terapéutica, que se sigue distribuyendo en función de 5 franjas de concentraciones de colesterol LDL y 4 niveles de RCV. Sin embargo, en la tabla de recomendaciones del control de lípidos, simplifica los niveles de riesgo a tres (muy alto riesgo, alto riesgo, y el resto) y no hace mención a enfermedades específicas, por lo que merece la pena recordar que casi todos los ictus isquémicos o el ataque isquémico transitorio tiene un RCV muy alto $^{74}$.

Si bien los objetivos terapéuticos en colesterol LDL para las tres principales categorías de RCV no han variado, para los pacientes de riesgo muy alto el objetivo alternativo a un colesterol $\mathrm{LDL}<70 \mathrm{mg} / \mathrm{dl}$ (1,8 mmol/L) puede ser una reducción del mismo de por lo menos el $50 \%$ si las concentraciones basales sin tratamiento están entre 70 y $135 \mathrm{mg} / \mathrm{dl}(1,8 \mathrm{mmol} / \mathrm{L} \mathrm{y} 3,5 \mathrm{mmol} / \mathrm{L})^{75}$. En los de riesgo alto, el objetivo es cLDL $<100 \mathrm{mg} / \mathrm{dl}(<2,6 \mathrm{mmol} / \mathrm{L})$ o una reducción de al menos el 50\%, si el basal está entre 100 y $200 \mathrm{mg} / \mathrm{dL}$ (2,6 y $5,1 \mathrm{mmol} / \mathrm{L})$. Se reconoce que los niveles bajos de colesterol HDL se asocian con un mayor RCV, pero que las estrategias para aumentar el colesterol HDL no se acompañan de una disminución del RCV.

En el apartado de la determinación del perfil lipídico, se recomienda utilizar el colesterol no HDL o la apoB como objetivos terapéuticos alternativos en presencia de hipertrigliceridemia. Dado que el colesterol total y HDL pueden medirse adecuadamente en muestras sin ayunas, el colesterol no HDL puede calcularse con facilidad e independientemente de la trigliceridemia. Ello de alguna forma está alineado con las directrices de un reciente documento de consenso en el que se expone que las determinaciones del perfil lipídico en ayunas o no ayunas deben ser complementarias y no excluyentes entre sí ${ }^{76}$.

Por lo que respecta al tratamiento farmacológico, las estatinas siguen siendo los fármacos de primera elección. La terapia de combinación estatina más ezetimiba también aporta beneficios cardiovasculares, datos que están en consonancia con el metanálisis del Cholesterol Treatment Trialists' Collaboration ${ }^{77}$ y apoyan el argumento de que la reducción del colesterol LDL es clave, independientemente del método utilizado. Finalmente, menciona la eficacia hipolipemiante de los nuevos anticuerpos monoclonales humanizados que inactivan la proproteína convertasa subtilisinakexina tipo 9 (PCSK9), a la espera de los resultados de estudios clínicos de morbi-mortalidad actualmente en curso. 


\section{Diabetes mellitus}

La ECV es la principal causa de morbimortalidad en personas con diabetes, una condición que confiere por sí misma un RCV elevado, y en la mayoría de los pacientes alto o muy alto. La excepción serían aquellos pacientes con diabetes tipo 2 de $<10$ años de evolución, sin otros factores de RCV y sin complicaciones, y los pacientes con diabetes tipo 1 jóvenes de corta evolución y sin complicaciones. En sujetos con diabetes, las ecuaciones de riesgo tienen un uso muy limitado en el cálculo del RCV y con frecuencia no han sido validadas $^{78}$.

Los pacientes con diabetes suelen presentar varios factores de RCV. La estrategia más eficaz es la intervención terapéutica multifactorial e integral, con objetivos de control para cada factor de riesgo modificable. El control de la HTA y la reducción de las concentraciones de colesterol reducen el RCV, y el control glucémico reduce significativamente el riesgo de complicaciones microvasculares y, en menor medida y a más largo plazo, de episodios macrovasculares ${ }^{79}$. Los cambios en el estilo de vida, mediante modificaciones sostenibles de la alimentación y el aumento de la actividad física que ayuden al control del peso corporal, son claves en el tratamiento de pacientes con diabetes tipo 2. En la mayoría de los pacientes con diabetes tipo 1 y tipo 2, se recomienda un objetivo de $\mathrm{HbA} 1 \mathrm{c}<7,0 \%(<53 \mathrm{mmol} / \mathrm{mol})^{80}$. En los pacientes con diabetes de larga evolución, edad avanzada, frágiles o con complicaciones graves, deben considerarse objetivos menos estrictos, especialmente si existe riesgo de hipoglucemia. Por el contrario, en los pacientes no frágiles con diagnóstico reciente o corta evolución de la diabetes tipo 2 debe considerarse un objetivo de HbA1c $\leq 6,5 \%$ $(\leq 48 \mathrm{mmol} / \mathrm{mol}$ ). Después de la metformina, la selección de los fármacos hipoglucemiantes debe ser individualizada, considerando el efecto sobre el riesgo de hipoglucemia y el peso, las preferencias del paciente y el coste. Tras la demostración del efecto beneficioso específico sobre el RCV de la empagliflozina y liraglutida en pacientes con diabetes tipo 2 de riesgo muy alto, la existencia de ECV debe favorecer la selección de estos fármacos cuando la metformina resulta insuficiente ${ }^{81,82}$.

Los fármacos hipolipemiantes, principalmente estatinas, se recomiendan en todos los pacientes con diabetes tipo 2 o tipo 1 de edad $>40$ años, y en los de $<40$ años con RCV elevado por la presencia de múltiples factores de riesgo o de complicaciones microvasculares ${ }^{77}$. En general el objetivo de presión arterial es $<140 / 85 \mathrm{~mm} \mathrm{Hg}$, y $130 / 80 \mathrm{~mm} \mathrm{Hg}$ en diabetes tipo 1, pero en los pacientes más jóvenes con riesgo elevado de complicaciones se recomienda $<130 / 80 \mathrm{~mm} \mathrm{Hg}^{83}$. El tratamiento antiagregante no está recomendado en los pacientes sin ECV.

\section{Hipertensión arterial}

Se recomienda la medida clínica de la presión arterial (PA) como cribado y diagnóstico de HTA, el cual debe ser basado en al menos dos medidas de la PA en cada visita, en al menos dos visitas. Si la PA está solo ligeramente elevada: HTA grado 1 (PA sistólica 140$159 \mathrm{~mm} \mathrm{Hg}$ y o PA diastólica $90-99 \mathrm{~mm} \mathrm{Hg}$ ), se recomienda repetir las medidas en varias ocasiones a lo largo de varios meses. Las medidas ambulatorias de la PA mediante MAPA o mediante automedida (AMPA) se correlacionan más estrechamente con el pronóstico y la lesión de órganos diana que la medida clínica, por lo que su uso es altamente recomendado, no solo como diagnóstico en sujetos no tratados, sino también para monitorizar los efectos del tratamiento y la mejora de la adherencia.

En la evaluación diagnóstica de los sujetos hipertensos se recomienda las siguientes pruebas de laboratorio: 1) hemoglobina; 2) una determinación en ayunas de glucemia, colesterol total, colesterol HDL, colesterol LDL, triglicéridos, ionograma, acido úrico, creatinina y filtrado glomerular estimado, y TSH en mujeres postmenopáusicas; y 3) un análisis de orina, con tira reactiva y cociente albuminuria/creatinina urinaria. Debe solicitarse un ECG al inicio, por la información pronóstica que puede aportar a pesar de su limitada sensibilidad, y en el seguimiento, dado el valor pronóstico de los cambios del ECG. La ecocardiografía y el fondo de ojo se considerarán en casos seleccionados. No se recomienda la determinación rutinaria de otros biomarcadores o pruebas de imagen vascular.

La decisión de iniciar el tratamiento farmacológico dependerá no solo del nivel de PA, sino también del RCV. La lesión subclínica de órganos diana, incluyendo la presencia de una excreción urinaria de albúmina $\geq 30 \mathrm{mg} / \mathrm{g}$ predice la mortalidad cardiovascular de forma independiente a la puntuación del SCORE, especialmente en el grupo de riesgo moderado. El tratamiento antihipertensivo puede ser considerado en los sujetos con HTA grado 1 y grado 2 (PA sistólica de $160-179 \mathrm{~mm} \mathrm{Hg}$ y o PA diastólica $100-109 \mathrm{~mm} \mathrm{Hg}$ ) de bajo riesgo, sin lesión de órganos diana, después de medidas repetidas de la PA y o confirmación de la elevación de la PA mediante medidas ambulatorias (MAPA o AMPA), si no se controla la PA con cambios favorables de estilo de vida, aunque en algunas situaciones (especialmente $\mathrm{n}$ la HTA grado 1 de bajo riesgo), el número necesario a tratar (NNT) puede ser elevado y se deben tener en cuenta las preferencias de cada paciente.

El principal beneficio del tratamiento antihipertensivo deriva de la reducción de la PA, independientemente del fármaco utilizado, pudiendo ser iniciado el tratamiento con diuréticos tiazídicos, o tipo tiazidas (indapamida, clortalidona), IECAS o ARA2, antagonistas del calcio o betabloqueantes. La posición de estos últimos como fármacos de primera línea ha sido cuestionada, debido a su menor efectividad en la prevención del ictus o sus posibles efectos metabólicos (aunque esto puede no ser extensible a todos los betabloqueantes), excepto en las situaciones clínicas con indicación específica de estos agentes (enfermedad coronaria, insuficiencia cardiaca con fracción de eyección reducida, etc.). Se recomienda evitar la asociación de betabloqueantes con diuréticos tiazídicos, especialmente en hipertensos con alteraciones metabólicas. Los IECA o ARA 2 estarían especialmente indicados en hipertensos con hipertrofia ventricular izquierda, microalbuminuria o proteinuria, para preservar la función renal y retrasar la evolución de la enfermedad renal crónica. Se preferirán los fármacos antihipertensivos con eficacia mantenida durante las 24 horas, y se recomienda simplificar el tratamiento para mejorar la adherencia.

En la mayoría de pacientes será necesario un tratamiento combinado con al menos dos fármacos, y en un 15-20\% de 3 o más. En cuanto al objetivo terapéutico, se recomienda una reducción de la $\mathrm{PA}<140 / 90 \mathrm{~mm} \mathrm{Hg}$ en todos los pacientes hipertensos y una PA sistólica $<150 \mathrm{~mm} \mathrm{Hg}$ en octogenarios. Parece prudente aconsejar una reducción de la PA entre 130-139 mm Hg de PA sistólica y una PA diastólica entre $80-85 \mathrm{~mm} \mathrm{Hg}$, posiblemente más cerca de $130 / 80 \mathrm{~mm} \mathrm{Hg}$ que de $140 / 90 \mathrm{~mm} \mathrm{Hg}$. Los resultados del estudio SPRINT y de metanálisis recientes sugieren que en algunos casos, y a criterio clínico, podría intensificarse aún más el tratamiento, valorando el riesgo/beneficio individual y el coste general de dicha intervención $^{84-86}$. Esto es aplicable a hipertensos de riesgo elevado, mientras que datos más recientes del estudio HOPE-3, muestran que el beneficio del tratamiento antihipertensivo en sujetos de riesgo intermedio solo se observa en los que presentaban cifras de PA elevadas (PA sistólica > $140 \mathrm{~mm} \mathrm{Hg})^{87,88}$.

\section{Tratamiento antiplaquetario}

\section{Para personas sin ECV}

Las guías europeas siguen sin recomendar la utilización de fármacos antiplaquetarios en prevención primaria por el riesgo aumentado de hemorragias. Tampoco estaría indicada la combinación de aspirina con clopidogrel. Las recomendaciones europeas 
no se plantean lo sugerido por las guías de USPSTF de iniciar el tratamiento antiplaquetario a partir de un RCV superior al 10\% ${ }^{89}$. El tratamiento a partir de este nivel de riesgo podría compensar el riesgo de hemorragia y obtener un beneficio neto que justificaría la indicación en prevención primaria.

\section{Para personas con ECV o cerebrovascular}

En la fase aguda del síndrome coronario agudo se recomienda aspirina asociada a un inhibidor de la P2Y12 (clopidogrel, prasugrel o ticagrelor) durante 1 año. Posteriormente, en la fase crónica después de un infarto de miocardio (más de 12 meses) se utilizaría la aspirina sola. Sin embargo, no comenta en el cuadro de las recomendaciones el tratamiento con clopidogrel en caso de intolerancia o alergia a la aspirina, aunque en el texto hace referencia al estudio CAPRIE, publicado hace 10 años, cuyos datos confirmarían esta posibilidad ${ }^{90}$. Por otro lado, no recomienda el prasugrel para pacientes con enfermedad coronaria estable, ni el ticagrelor si no han tenido un síndrome coronario agudo. En el accidente isquémico transitorio o enfermedad cerebrovascular aguda no cardioembólica se recomienda la aspirina sola o asociada al dipiridamol, o clopidogrel solo.

\section{Adherencia al tratamiento}

La adherencia a la medicación de los individuos en alto riesgo y los pacientes con ECV es baja. Se recomienda simplificar el régimen terapéutico y que los médicos evalúen la adherencia al tratamiento e identifiquen las causas de la falta de adherencia para adaptar mejor las intervenciones futuras. Como novedad, destaca el análisis del policomprimido, es decir, la combinación a dosis fijas de una estatina, un fármaco antihipertensivo, aspirina y, en ocasiones, folato. En general, los resultados de un metaanálisis y una revisión sistemática no encontraron evidencia convincente del beneficio cardiovascular de esta estrategia frente a la multifarmacológica, pero sí se obtuvo una mejoría de la adherencia.

\section{¿Cómo intervenir a nivel poblacional?}

\section{Promover un estilo de vida saludable}

Según el paradigma de Geoffrey Rose, cambios pequeños en el RCV de toda la población producen reducciones de la carga de enfermedad mayores que grandes cambios únicamente en personas de alto riesgo. Este enfoque poblacional proporciona una serie de beneficios adicionales: aborda la salud cardiovascular durante toda la vida, previene otras comorbilidades, reduce las desigualdades sociales en salud, ahorra costes sanitarios y laborales, y mejora la esperanza y calidad de vida ${ }^{9}$. La conducta individual viene determinada por un entorno con diversos niveles jerárquicos: la familia, el grupo cultural y étnico, el lugar de trabajo, la atención sanitaria, las políticas nacionales e internacionales, que condicionan e influyen poderosamente en las elecciones personales.

Los profesionales de la salud pueden ejercer un papel importante en la promoción de intervenciones poblacionales, proporcionando a los grupos de apoyo, comunidades y políticos locales y nacionales, propuestas basadas en la evidencia sobre intervenciones eficaces dirigidas a cambiar el entorno para hacer fáciles las elecciones saludables.

\section{Dieta}

Los cambios en la dieta poblacional se acompañan de reducciones rápidas de los episodios cardiovasculares ${ }^{91}$. El reconocimiento de los beneficios para la salud de la reducción del aporte energético y el contenido de sal, azúcar y grasas trans y saturadas, ha llevado en algunos países europeos a tomar medidas exitosas de reducción de su contenido en alimentos y bebidas ${ }^{92}$. El establecimiento de límites superiores armonizados en la Unión Europea aseguraría el mismo nivel de protección para todos los consumidores europeos ${ }^{93}$.

La limitación de la exposición de los niños a la publicidad de alimentos y bebidas no saludables es una de las medidas de prevención de la obesidad infantil más coste-efectivas ${ }^{94}$. En España, esta materia la regula el código PAOS, que se ha mostrado incapaz de limitar la exposición de los niños a la publicidad de alimentos y bebidas no saludables ${ }^{95}$, por lo que es preciso instaurar un sistema de protección eficaz, que evite la exposición de los menores a este tipo de publicidad. Además, la mayoría de productos anunciados en España con declaraciones de salud o nutricionales tampoco son saludables, pudiendo inducir a confusión a los niños y sus padres ${ }^{96}$, por lo que este tipo de alegaciones deberían regularse, impidiendo que pueda hacerse uso de las mismas en alimentos y bebidas no saludables.

En un estudio reciente con consumidores españoles, el 80\% de los que leían el etiquetado nutricional lo hacían para elegir productos saludables ${ }^{97}$. La Red Europea del Corazón reclama un esquema simplificado, basado en códigos de color, en la parte frontal del envase, que indique la composición de nutrientes de nivel alto, medio o bajo $^{91}$. Este método (semáforo nutricional múltiple), que se puede aplicar a todos los alimentos y extenderse a determinados restaurantes, es el más eficaz para ayudar a los consumidores a elegir productos saludables $^{98}$.

Las estrategias de precios pueden conducir a una disminución de las ventas de alimentos no saludables y a un aumento de las ventas de frutas y verduras, mejorando el consumo de energía y nutrientes, el IMC y la salud ${ }^{99}$. El impuesto a la grasa en Dinamarca originó una disminución de su consumo de un 10-15\% y el impuesto a la comida basura en Hungría hizo que las ventas cayeran un $27 \%{ }^{100}$. El impuesto a las bebidas azucaradas en México provocó un descenso en las compras del $12 \%$, con mayor intensidad en los hogares de nivel socioeconómico bajo, donde alcanzó el 17\%101. En la tabla 1, con datos de la Encuesta Nacional de Salud, se observa un gradiente social muy intenso en el consumo diario de refrescos entre los menores españoles. Al comparar las dos encuestas más recientes ${ }^{102,103}$, se constata que tanto el consumo de estos productos como el gradiente social asociado al mismo disminuyeron en 2012 con respecto a 2006, probablemente por efecto de la crisis económica. Esta hipótesis es consistente con la alta elasticidad al precio observada para el consumo de bebidas azucaradas, que coloca el impuesto a este tipo de bebidas como un política con potencial para combatir la obesidad infantil en España y reducir el gradiente social asociado a la misma, pues el impuesto sería más efectivo en los consumidores de renta baja, que son los más elásticos al precio ${ }^{104}$. En cualquier caso, los incentivos económicos (impuestos y subsidios) deben equilibrarse para contrarrestar un posible impacto negativo en los grupos socialmente desfavorecidos.

Para hacer frente a la obesidad, todas las escuelas y los centros de trabajo deben tener protocolos dirigidos a promocionar un entorno saludable y ofrecer alimentos sanos ${ }^{100}$. Las intervenciones para modificar la dieta en los centros de trabajo ${ }^{105}$, el establecimiento de estándares alimentarios y nutricionales, y la prohibición de la venta de bebidas azucaradas y alimentos no saludables en los colegios, son medidas eficaces para promover patrones de consumo más saludables ${ }^{106}$. Al objeto de promover una dieta equilibrada, asegurando un entorno alimentario adecuado, el Consejo Interterritorial del Sistema Nacional de Salud aprobó en 2010 el Documento de Consenso sobre la Alimentación en los Centros Educativos, con recomendaciones sobre estándares alimentarios y nutricionales para la oferta alimentaria en comedores, cafeterías y máquinas expendedoras ${ }^{107}$. En 2011, la Ley de Seguridad Alimentaria y Nutrición, en su artículo 40, estableció que no se permitirá la venta de alimentos y bebidas con un alto contenido en ácidos grasos 
Tabla 1

Prevalencia de consumo diario de refrescos en españoles de 5 a 14 años, por clase social

\begin{tabular}{|c|c|c|c|c|}
\hline \multirow[t]{2}{*}{ Consumo diario } & \multicolumn{2}{|c|}{ ENSE 2006-7 } & \multicolumn{2}{|c|}{ ENSE 2011-12 } \\
\hline & \% (IC95\%) & $\mathrm{n}$ & \% (IC95\%) & $\mathrm{n}$ \\
\hline \multicolumn{5}{|l|}{ Refrescos } \\
\hline Alta & $8,9(7,4-10,5)$ & 113 & $6,5(4,7-8,3)$ & 49 \\
\hline Media & $17,7(16,4-19,0)$ & 601 & $10,8(9,5-12,1)$ & 233 \\
\hline Baja & $31,7(28,2-35,3)$ & 210 & $16,7(13,3-20,2)$ & 76 \\
\hline
\end{tabular}

Fuente: Elaboración propia a partir de los datos de la ENSE (Encuesta Nacional de Salud) ${ }^{102,103}$.

saturados, ácidos grasos trans, sal y azúcares en las escuelas infantiles $\mathrm{y}$ en los centros escolares ${ }^{108}$. No obstante, en un estudio realizado en 2015 , la inmensa mayoría de los productos ofertados en las máquinas expendedoras de los centros de secundaria de la Comunidad de Madrid fueron no saludables ${ }^{109}$. Por ello, urgimos a las autoridades a establecer reglamentariamente los criterios nutricionales de alimentos y bebidas, para evitar la venta de productos no saludables, dando cumplimiento a lo estipulado en la ley.

\section{Actividad fisica}

El sedentarismo y la inactividad física afectan a más de la mitad de la población. La actividad física regular se recomienda durante toda la vida como parte de un estilo de vida saludable. Cualquier actividad es mejor que nada, pero se recomienda al menos 150 minutos/semana de actividad moderada ó 75 minutos/semana de actividad vigorosa o una combinación equivalente ${ }^{110}$. El problema del sedentarismo no empieza en la edad adulta. La educación y promoción de la actividad física deben empezar en edad preescolar, cifrándose a cantidad mínima diaria en la escuela de al menos 30 minutos y preferiblemente de 60 minutos.

El hábito de un estilo de vida activo debe empezar en edades tempranas y debe incluir escuelas, familias, comunidades y entidades gubernamentales. La creación de infraestructuras en barrios y comunidades que garanticen un ambiente más seguro (carriles bicicleta, zonas peatonales, parques, etc.) incentivarán la actividad física cotidiana. A modo de ejemplo, se proponen las siguientes estrategias simples para aumentar la actividad física diaria:

Fomentar el uso de las escaleras en lugar del ascensor o las escaleras mecánicas, mediante señalización para dirigir a las personas a las escaleras y divulgación de materiales de promoción de la salud que informen de los efectos positivos de subiré escaleras.

Incrementar los precios del combustible para reducir el uso de automóviles y aumentar el desplazamiento activo a pie o en bicicleta.

Asegurar la continuidad de la educación en el estilo de vida activo desde la edad preescolar, pasando por todos los niveles de educación primaria y secundaria. Insistir en que la actividad física también mejora el rendimiento escolar ${ }^{111}$. Fomentar el desplazamiento a los centros escolares "a pie".

Ofrecer en los lugares de trabajo diferentes opciones para la promoción de la actividad física ${ }^{112}$.

\section{Tabaquismo}

Las siguientes medidas son eficaces para promover el abandono del tabaquismo: el empaquetado sencillo, las restricciones a la publicidad, promoción y patrocinio de la industria tabacalera113, la prohibición de su consumo en lugares públicos, incluido los puesto de trabajo, y la prohibición de su venta a adolescentes y su venta en máquinas expendedoras ${ }^{114}$. Además, para reducir el uso del tabaco se debe mantener elevados los impuestos en todos los productos de tabaco, recomendar a las mujeres embarazadas no fumar, a los profesionales sanitarios, cuidadores y profesores no consumir tabaco en horario laboral, y a los padres no fumar en presencia de niños. Además, las campañas educativas en las escuelas reducen el inicio del uso del tabaco y promueven el abandono tanto en los niños como en el profesorado ${ }^{114}$.

\section{Protección contra el abuso de alcohol}

A escala poblacional, el consumo de alcohol se asocia a múltiples riesgos para la salud que sobrepasan cualquier potencial efecto beneficioso. En 2012, el 5,9\% de todas las muertes (1/3 de las mismas por ECV) y el 5,1\% de la carga total de enfermedad en años de vida ajustados por discapacidad fueron atribuibles al consumo de alcohol. La mortalidad por cardiopatía isquémica es más del doble en varones y mujeres muy bebedores ${ }^{115}$. Sin embargo, un consumo de alcohol ligero (1-3 unidades/día) en algunos segmentos de la población se ha asociado a menor mortalidad coronaria ${ }^{116}$.

Las estrategias con mayor nivel de eficacia para prevenir el consumo de alcohol son: límites de edad para la venta y el suministro de alcohol, control de alcoholemia en conductores, control de la venta minorista y reducción de los horarios de apertura, prohibición del alcohol en la publicidad, la promoción y el patrocinio de eventos, y aumento de los precios de venta. Todas estas intervenciones son rentables y coste-efectivas.

\section{Entorno saludable}

La Comisión Europea ha propuesto medidas a implementar en 2030, dirigidas a reducir las emisiones perjudiciales procedentes del tráfico, las plantas de producción energética y la agricultura. Se deben impulsar esfuerzos y medidas encaminadas a disminuir la contaminación del aire, que deberían asumir los gobiernos nacionales a través de una legislación adecuada y eficaz ${ }^{117}$. Las organizaciones de pacientes y los profesionales sanitarios deben hacer oír su voz en las iniciativas educativas y políticas necesarias para lograr los objetivos mencionados. Individualmente, se debe informar al paciente sobre qué hacer durante los periodos de contaminación.

\section{¿Dónde intervenir a nivel individual?}

\section{Atención primaria}

La prevención cardiovascular se debe implementar en todos los niveles del sistema sanitario y de la sociedad en general. Se recomienda que los médicos de familia, enfermería y otros profesionales sanitarios de atención primaria desempeñen actividades de prevención cardiovascular en pacientes de riesgo alto. Se recomienda un abordaje sistemático para evaluar el RCV, dando prioridad a las personas de riesgo alto, y no se recomienda el cribado oportunista en personas menores de 40 años de edad sin factores de riesgo.

Existen evidencias del éxito del papel de enfermería conjuntamente con el médico de familia en la mejora del control de los factores de RCV, cuando se compara con la atención habitual ${ }^{118}$. 


\section{Atención hospitalaria}

Para reducir la morbilidad y la mortalidad después de un episodio agudo, se recomienda implementar estrategias de prevención cardiovascular incluyendo cambios en los estilos de vida y en el control de los factores de riesgo antes de dar el alta de la hospitalización.

\section{Rehabilitación cardíaca}

Para reducir la recurrencia de la enfermedad en pacientes estables con ECV, se recomiendan los programas preventivos dirigidos a la optimización terapéutica, la adherencia y el control de los factores de riesgo. La rehabilitación cardíaca es un programa integral que incluye ejercicio físico, modificación de los factores de riesgo, educación y apoyo psicológico. Una revisión sistemática concluyó que, para pacientes con riesgo bajo-moderado que tienen insuficiencia cardíaca o que hayan tenido un infarto de miocardio o revascularización, la rehabilitación cardíaca basada en el ejercicio físico disminuye los ingresos hospitalarios y mejora la calidad de vida relacionada con la salud en comparación con la atención habitual, y puede reducir la mortalidad a largo plazo ${ }^{119}$.

El estudio RESPONSE aleatorizó a pacientes con síndrome coronario agudo a recibir atención convencional o un programa de prevención de visitas ambulatorias coordinado por enfermeras durante 6 meses $^{120}$. Al cabo de 1 año, los pacientes del grupo del programa de prevención tenían mejor control de los factores de riesgo, menores tasas de reingresos hospitalarios, menos visitas a urgencias y una reducción del RCV de un $17 \%$ en comparación con el grupo control.

Las nuevas tecnologías como la telerehabilitación o las aplicaciones para móviles pueden aumentar aún más la participación y ofrecer más apoyo conductual y monitorización individualizados, aunque hacen falta estudios clínicos aleatorizados a gran escala. Los estándares del cumplimiento en la prevención de la ECV pueden servir como vehículos para acelerar la traslación adecuada de la evidencia científica a la práctica clínica.

\section{¿Dónde intervenir a nivel poblacional?}

Las intervenciones poblacionales para promover la salud cardiovascular y hacer frente a las dietas poco saludables, el tabaquismo y la inactividad física, deben realizarse desde el ámbito internacional, nacional, regional y local. A nivel nacional, en España existen iniciativas como las estrategias en cardiopatía isquémica, ictus o diabetes del Sistema Nacional de Salud ${ }^{121-123}$, que suponen un esfuerzo consensuado entre el Ministerio de Sanidad, las Comunidades Autónomas, las sociedades científicas, las asociaciones de pacientes y los profesionales expertos. Además, se dispone de la Estrategia para la Nutrición, Actividad Física y Prevención de la Obesidad (NAOS) para mejorar los hábitos alimentarios y aumentar la práctica regular de la actividad física de todos los ciudadanos, con especial énfasis en la población infantil ${ }^{124}$. A nivel regional, las Comunidades Autónomas disponen de programas que orientan sus actuaciones en prevención cardiovascular $^{125}$. Quince sociedades científicas involucradas en la prevención cardiovascular con presencia en todas las Comunidades Autónomas y dos representantes de la administración constituyen el CEIPC, que se encarga de adaptar, difundir e implementar las Guías Europeas de Prevención Cardiovascular ${ }^{2}$.

Desde la Unión Europea y los gobiernos nacionales, se debería legislar, por ejemplo, sobre la publicidad alimentaria dirigida a niños, la composición y el etiquetado nutricional de los alimentos, las políticas y los ambientes libres de humo, la comercialización de los alimentos poco saludables, bebidas alcohólicas y tabaco, y la promoción de entornos que fomenten la actividad física cotidiana.
Además, se deben desarrollar medidas para reducir la contaminación del aire. Para apoyar las estrategias de alimentación y nutrición, tabaco y alcohol, se pueden utilizar medidas económicas como los impuestos y los subsidios. El garantizar la disponibilidad y accesibilidad a la actividad física y los alimentos saludables debería ser un esfuerzo conjunto del gobierno, las empresas y los comercios. Las autoridades sanitarias deben supervisar los avances conseguidos, y si los esfuerzos que voluntariamente realiza la industria son inadecuados, los gobiernos deben intervenir.

Los profesionales de la salud y las organizaciones no gubernamentales tienen la responsabilidad de establecer la agenda y el seguimiento de las intervenciones para promover la prevención cardiovascular. Por su parte, las organizaciones de pacientes cardiovasculares informan y ayudan a los pacientes, y promueven la rehabilitación cardiaca.

En la creación de ambientes sanos y activos, especialmente en las escuelas, lugares de trabajo y comunidad, pueden desempeñar un papel importante las partes interesadas: profesores y asociaciones de padres, el sector de la restauración, organizaciones empresariales, sindicatos, clubes deportivos y gimnasios, y las organizaciones que participan en la planificación urbana y la movilidad, promoviendo el transporte público a pie o en bicicleta.

\section{Conflicto de intereses}

Los autores declaran no tener ningún conflicto de intereses.

\section{Bibliografía}

1. Piepoli MF, Hoes AW, Agewall S, Albus C, Brotons C, Catapano AL, et al. European guidelines on cardiovascular disease prevention in clinical practice. Eur Heart J. 2016; . 2016;37:2315-81.

2. Royo-Bordonada MA, Lobos Bejarano JM, Villar Alvarez F, Sans S, Pérez A, PedroBotet J, et al. Comentarios del Comité Español Interdisciplinario de Prevención Cardiovascular (CEIPC) a las guías europeas de prevención cardiovascular 2012. Rev Esp Salud Pública. 2013:87:103-20.

3. Brotons C, Royo-Bordonada MA, Alvarez-Sala L, Armario P, Artigao R, Conthe $\mathrm{P}$, et al. Adaptación española de la guía europea de prevención cardiovascular. Rev Esp Salud Pública. 2004;78:435-8.

4. Lobos JM, Royo-Bordonada MA, Brotons C, Álvarez-Sala L, Armario P, Maiques A, et al. Guía europea de prevención cardiovascular en la práctica clínica. Adaptación española del CEIPC 2008. Rev Esp Salud Pública. 2008;82:581-616.

5. A Dictionary of Epidemiology. 4 th ed. New York: Oxford University Press.

6. Instituto Nacional de Estadística. Defunciones según la Causa de Muerte 2014. Tablas Nacionales. Defunciones por causas (lista reducida), sexo y edad. Disponible en: http://www.ine.es/dyngs/INEbase/es/operacion.htm?c= Estadistica_C\&cid=1254736176780\&menu=ultiDatos\&idp $=1254735573175$

7. Villar F, Banegas JR, Donado J de Mata, Rodríguez Artalejo F. Las enfermedades cardiovasculares y sus factores de riesgo en España: hechos y cifras. Informe SEA 2007. Sociedad Española de Arteriosclerosis.

8. Flores-Mateo G, Grau M, O'Flaherty M, Ramos R, Elosua R, Violan-Fors C, et al. Análisis de la disminución de la mortalidad por enfermedad coronaria en una población mediterránea: España 1998-2005. Rev Esp Cardiol. 2011;64:988-96.

9. National Institute for Health and Clinical Excellence. Cardiovascular disease prevention. Public health guideline [PH 25]. NICE 2010. http://www.nice. org.uk/guidance/PH25

10. Kotseva K, Wood D, De Bacquer D, De Backer G, Ryden L, Jennings C, et al EUROASPIRE IV: a European Society of Cardiology survey on the lifestyle, risk factor and therapeutic management of coronary patients from 24 European countries. Eur J Prev Cardiol. 2016;23:636-48

11. Marrugat J, Vila J, Baena-Díez JM, Grau M, Sala J, Ramos R, et al. Validez relativa de la estimación del riesgo cardiovascular a 10 años en una cohorte poblacional del estudio REGICOR. Rev Esp Cardiol. 2011;64:385-94.

12. Rose G. Sick individuals and sick populations. Int J Epidemiol. 1985;14:32-8.

13. Agüero F, Dégano IR, Subirana I, Grau M, Zamora A, Sala J, et al. Impact of a partial smoke-free legislation on myocardial infarction incidence, mortality and case-fatality in a population-based registry: the REGICOR Study. PLoS One. 2013;8:e53722.

14. Cobiac LJ, Magnus A, Lim S, Barendregt JJ, Carter R, Vos T. Which interventions offer best value for money in primary prevention of cardiovascular disease? PLoS One. 2012; 7:e41842.

15. Mistry H, Morris S, Dyer M, Kotseva K, Wood D, Buxton M. Cost-effectiveness of a European preventive cardiology programme in primary care: a Markov modelling approach. BMJ Open. 2012;2:e001029.

16. Plans-Rubio P. The cost effectiveness of statin therapies in Spain in 2010 after the introduction of generics and reference prices. Am J Cardiovasc Drugs. 2010;10:369-82 
17. Mason H, Shoaibi A, Ghandour R, O'Flaherty M, Capewell S, Khatib R, et al. A cost effectiveness analysis of salt reduction policies to reduce coronary heart disease in four Eastern Mediterranean countries. PLoS One. 2014;9:e84445.

18. O'Keeffe C, Kabir Z, O'Flaherty M, Walton J, Capewell S, Perry IJ. Modelling the impact of specific food policy options on coronary heart disease and stroke deaths in Ireland. BMJ Open. 2013;3:e002837.

19. Pereira M, Azevedo A, Lunet N, Carreira H, O'Flaherty M, Capewell S, et al. Explaining the decline in coronary heart disease mortality in Portugal between 1995 and 2008. Circ Cardiovasc Qual Outcomes. 2013;6:634-42.

20. Banegas JR, Graciani A, de la Cruz-Troca JJ, León-Muñoz LM, Guallar-Castillón $\mathrm{P}$, Coca A, et al. Achievement of cardiometabolic goals in aware hypertensive patients in Spain. A nationwide population-based study. Hypertension. 2012;60:898-905.

21. Guallar-Castillón P, Gil-Montero M, León-Muñoz LM, Graciani A, Bayán-Bravo A, Taboada JM, et al. Magnitude and management of hypercholesterolemia in the adult population of Spain, 2008-2010: The ENRICA Study. Rev Esp Cardiol. 2012;65:551-8.

22. Grau M, Elosua R, Cabrera de León A, Guembe MJ, Baena-Díez JM, Vega Alonso $\mathrm{T}$, et al. Factores de riesgo cardiovascular en España en la primera década del siglo XXI: análisis agrupado con datos individuales de 11 estudios de base poblacional, estudio DARIOS. Rev Esp Cardiol. 2011;64:295-304.

23. Sundstrom J, Arima H, Woodward M, Jackson R, Karmali K, Lloyd-Jones D, et al., Blood Pressure Lowering Treatment Trialists' Collaboration. Blood pressurelowering treatment based on cardiovascular risk: a meta-analysis of individual patient data. Lancet. 2014:384:591-8.

24. Mihaylova B, Emberson J, Blackwell L, Keech A, Simes J, Barnes EH, et al., Cholesterol Treatment Trialists' (CTT) Collaborators. The effects of lowering LDL cholesterol with statin therapy in people at low risk of vascular disease: meta-analysis of individual data from 27 randomised trials. Lancet. 2012;380:581-90.

25. Sans S, Fitzgerald AP, Royo D, Conroy R, Graham I. Calibración de la tabla SCORE de riesgo cardiovascular para España. Rev Esp Cardiol. 2007:60:476-85.

26. Marrugat J, Subirana I, Ramos R, Vila J, Marín-Ibañez A, Guembe MJ, et al. Derivation and validation of a set of 10-year cardiovascular risk predictive functions in Spain: the FRESCO Study. Prev Med. 2014;61:66-74.

27. Gabriel R, Brotons C, Tormo MJ, Segura A, Rigo F, Elosua R, et al. The ERICE-score: the new native cardiovascular score for the low-risk and aged Mediterranean population of Spain. Rev Esp Cardiol. 2015;68:205-15.

28. Marrugat J, Subirana I, Comín E, Cabezas C, Vila J, Elosua R, et al. Validity of an adaptation of the Framingham cardiovascular risk function: the VERIFICA Study. J Epidemiol Community Health. 2007;61:40-7.

29. Royo-Bordonada MÁ, Lobos JM, Brotons C, Villar F, de Pablo C, Armario P, et al. The condition of the cardiovascular prevention in Spain. Med Clin (Barc). 2014;142:7-14.

30. D'Agostino RB Sr, Vasan RS, Pencina MJ, Wolf PA, Cobain M, Massaro JM, et al. General cardiovascular risk profile for use in primary care: the Framingham Heart Study. Circulation. 2008;117:743-53.

31. Cuende JI, Cuende N, Calaveras-Lagartos J. How to calculate vascular age with the SCORE project scales: a new method of cardiovascular risk evaluation. Eur Heart J. 2010;31:2351-8.

32. Cooney MT, Selmer R, Lindman A, Tverdal A, Menotti A, Thomsen T, et al. Cardiovascular risk estimation in older persons: SCORE O.P. Eur J Prev Cardiol. 2016:23:1093-103.

33. Brotons C, Moral I, Fernández D, Cuixart L, Muñox A, Soteras A, et al. Clinical consequences of using the new cardiovascular risk tables SCORE OP in patients aged over 65 years. Med Clin (Barc). 2016;147:381-6.

34. Hlatky MA, Greenland P, Arnett DK, Ballantyne CM, Criqui MH, Elkind MS, et al. Criteria for evaluation of novel markers of cardiovascular risk: a scientific statement from the American Heart Association. Circulation. 2009;119: 2408-16.

35. Tzoulaki I, Siontis KC, Evangelou E, Ioannidis JP. Bias in associations of emerging biomarkers with cardiovascular disease. JAMA Intern Med. 2013;173:664-71.

36. Silber S. Comparison of spiral and electron beam tomography in the evaluation of coronary calcification in asymptomatic persons. Int J Cardiol. 2002;82:297-8.

37. Lorenz MW1, Polak JF, Kavousi M, Mathiesen EB, Völzke H, Tuomainen TP, et al., PROG-IMT Study Group. Carotid intima-media thickness progression to predict cardiovascular events in the general population (the PROG-IMT collaborative project): a meta-analysis of individual participant data. Lancet. 2012;379:2053-62.

38. Den Ruijter HM, Peters SA, Anderson TJ, Britton AR, Dekker JM, Eijkemans MJ, et al. Common carotid intima-media thickness measurements in cardiovascular risk prediction: a meta-analysis. JAMA. 2012;308:796-803.

39. Fowkes FG, Murray GD, Butcher I, Heald CL, Lee RJ, Chambless LE, et al., Ankle Brachial Index Collaboration. Ankle brachial index combined with Framingham Risk Score to predict cardiovascular events and mortality: a meta-analysis. JAMA. 2008;300:197-208.

40. Schiffrin EL, Lipman ML, Mann JF. Chronic kidney disease: effects on the cardiovascular system. Circulation. 2007;116:85-97.

41. Smeeth L, Thomas SL, Hall AJ, Hubbard R, Farrington P, Vallance P. Risk of myocardial infarction and stroke after acute infection or vaccination. N Engl J Med. 2004;351:2611-8.

42. Dietrich T, Jimenez M, Krall Kaye EA, Vokonas PS, Garcia RI. Age-dependent associations between chronic periodontitis/edentulism and risk of coronary heart disease. Circulation. 2008;117:1668-74.
43. Kalam K, Marwick TH. Role of cardioprotective therapy for prevention of cardiotoxicity with chemotherapy: a systematic review and meta-analysis. Eur J Cancer. 2013;49:2900-9.

44. Peters MJ, Symmons DP, McCarey D, Dijkmans BA, Nicola P, Kvien TK, et al. EULAR evidence-based recommendations for cardiovascular risk management in patients with rheumatoid arthritis and other forms of inflammatory arthritis. Ann Rheum Dis. 2010;69:325-31.

45. Gonzaga C, Bertolami A, Bertolami M, Amodeo C, Calhoun D. Obstructive sleep apnea, hypertension and cardiovascular diseases. J Hum Hypertens. 2015;29:705-12.

46. Vlachopoulos C, Jackson G, Stefanadis C, Montorsi P. Erectile dysfunction in the cardiovascular patient. Eur Heart J. 2013;34:2034-46.

47. World Health Organization. Familial hypercholesterolemia-report of a second WHO consultation. Geneva: World Health Organization; 1999.

48. Kutner JS, Blatchford PJ, Taylor DH Jr, Ritchie CS, Bull JH, Fairclough DL, et al. Safety and benefit of discontinuing statin therapy in the setting of advanced, life-limiting illness: a randomized clinical trial. JAMA Intern Med 2015:175:691-700.

49. Skjaerven R, Wilcox AJ, Klungsoyr K, Irgens LM, Vikse BE, Vatten LJ, et al. Cardiovascular mortality after pre-eclampsia in one child mothers: prospective, population based cohort study. BMJ. 2012;345:e7677.

50. Moran LJ, Misso ML, Wild RA, Norman RJ. Impaired glucose tolerance, type 2 diabetes and metabolic syndrome in polycystic ovary syndrome: a systematic review and meta-analysis. Hum Reprod Update. 2010;16:347-63.

51. Bhopal R, Fischbacher C, Vartiainen E, Unwin N, White M, Alberti G. Predicted and observed cardiovascular disease in South Asians: application of FINRISK, Framingham and SCORE models to Newcastle Heart Project data. J Public Health. 2005;27:93-100.

52. Rubak S, Sandbaek A, Lauritzen T, Christensen B. Motivational interviewing: a systematic review and meta-analysis. Br J Gen Pract. 2005;55:305-12.

53. Balady GJ, Williams MA, Ades PA, Bittner V, Comoss P, Foody JM, et al. American Heart Association Exercise CR. Core components of cardiacrehabilitation/secondary prevention programs: 2007 update: a scientific statement from the American Heart Association Exercise, Cardiac Rehabilitation, and Prevention Committee, the Council on Clinical Cardiology; the Councils on Cardiovascular Nursing, Epidemiology and Prevention, and Nutrition, Physical Activity, and Metabolism; and the American Association of Cardiovascular and Pulmonary Rehabilitation. Circulation. 2007;115:2675-82.

54. Whalley B, Thompson DR, Taylor RS. Psychological interventions for coronary heart disease: cochrane systematic review and meta-analysis. Int J Behav Med. 2014;21:109-21.

55. Rutledge T, Redwine LS, Linke SE, Mills PJ. A meta-analysis of mental health treatments and cardiac rehabilitation for improving clinical outcomes and depression among patients with coronary heart disease. Psychosom Med. 2013;75:335-49.

56. Stewart JC, Perkins AJ, Callahan CM. Effect of collaborative care for depression on risk of cardiovascular events: data from the IMPACT randomized controlled trial. Psychosom Med. 2014;76:29-37.

57. Sattelmair J, Pertman J, Ding EL, Kohl HW, Haskell W, Lee IM. Dose response between physical activity and risk of coronary heart disease: a meta-analysis. Circulation. 2011;124:789-95.

58. Lee DC, Pate RR, Lavie CJ, Sui X, Church TS, Blair SN. Leisure-time running reduces all-cause and cardiovascular mortality risk. J Am Coll Cardiol. 2014;64:472-81.

59. Marijon E, Tafflet M, Celermajer DS, Dumas F, Perier MC, Mustafic H, et al. Sports-related sudden death in the general population. Circulation. 2011;124:672-81.

60. Taylor T, Bryant LD, Keyse A, Joloza MT. Smoking-related behaviour and attitudes, 2005. London: Office for National Statistics; 2006.

61. He J, Vupputuri S, Allen K, Prerost MR, Hughes J, Whelton PK. Passive smoking and the risk of coronary heart disease-a meta-analysis of epidemiologic studies. N Engl J Med. 1999;340:920-6.

62. Cahill K, Stevens S, Perera R, Lancaster T. Pharmacological interventions for smoking cessation: an overview and network meta-analysis. Cochrane Database Syst Rev. 2013;5:CD009329.

63. Bullen C, Howe C, Laugesen M, McRobbie H, Parag V, Williman J, et al. Electronic cigarettes for smoking cessation: a randomised controlled trial. Lancet. 2013;382:1629.

64. Fernández-San Juan PM. Trans fatty acids (tFA): sources and intake levels, biological effects and content in commercial Spanish food. Nutr Hosp. 2009;24:515-20.

65. Mozaffarian D, Katan MB, Ascherio A, Stampfer MJ, Willett WC. Trans fatty acids and cardiovascular disease. N Engl J Med. 2006;354:1601-13.

66. Yang Q, Zhang Z, Gregg E, Flanders W, Merritt R, Hu F. Added sugar intake and cardiovascular diseases mortality among US adults. JAMA Intern Med. 2014;174:516-24.

67. Mente A, O’Donnell M, Rangarajan S, Dagenais G, Lear S, McQueen M. Associations of urinary sodium excretion with cardiovascular events in individuals with and without hypertension: a pooled analysis of data from four studies. Lancet. 2016;388:465-75.

68. de Koning L, Malik VS, Kellogg MD, Rimm EB, Willett WC, Hu FB. Sweetened beverage consumption, incident coronary heart disease, and biomarkers of risk in men. Circulation. 2012;125:1735-41.

69. Mozaffarian D. Dietary and policy priorities for cardiovascular disease, diabetes, and obesity: a comprehensive review. Circulation. 2016;133:187-225. 
70. Oreopoulos A, Padwal R, Norris CM, Mullen JC, Pretorius V, Kalantar-Zadeh K. Effect of obesity on short- and long-term mortality postcoronary revascularization: a meta-analysis. Obesity. 2008;16:442-50.

71. Berrington de Gonzalez A, Hartge P, Cerhan JR, Flint AJ, Hannan L, MacInnis RJ, et al. Body-mass index and mortality among 1.46 million white adults. N Engl J Med. 2010;363:2211-9.

72. Wormser D, Kaptoge S, Di Angelantonio E, Wood AM, Pennells L, Thompson A, et al., Emerging Risk Factors Collaboration. Separate and combined associations of body-mass index and abdominal adiposity with cardiovascular disease: collaborative analysis of 58 prospective studies. Lancet. 2011;377:1085-95.

73. Perk J, De Backer G, Gohlke H, Graham I, Reiner Z, Verschuren M, et al., European Association for Cardiovascular Prevention \& Rehabilitation (EACPR); ESC Committee for Practice Guidelines (CPG). European Guidelines on cardiovascular disease prevention in clinical practice (version 2012). The Fifth Joint Task Force of the European Society of Cardiology and Other Societies on Cardiovascular Disease Prevention in Clinical Practice (constituted by representatives of nine societies and by invited experts). Eur Heart J. 2012;33:1635-701.

74. Cannon CP, Blazing MA, Giugliano RP, McCagg A, White JA, Theroux P, et al., IMPROVE-IT Investigators. Ezetimibe added to statin therapy after acute coronary syndromes. N Engl J Med. 2015;372:2387-97.

75. Fuentes B, Gállego J, Gil-Núñez A, Morales A, Purroy F, Roquer J, et al., Comité ad hoc del Grupo de Estudio de Enfermedades Cerebrovasculares de la SEN. Guidelines for the preventive treatment of ischaemic stroke and TIA (I). Update on risk factors and life style. Neurología. 2012;27:560-74.

76. Nordestgaard BG, Langsted A, Mora S, Kolovou G, Baum H, Bruckert E, et al., European Atherosclerosis Society (EAS) and the European Federation of Clinical Chemistry and Laboratory Medicine (EFLM) Joint Consensus Initiative. Fasting is not routinely required for determination of a lipid profile: clinical and laboratory implications including flagging at desirable concentration cutpoints. A Joint Consensus Statement from the European Atherosclerosis Society and European Federation of Clinical Chemistry and Laboratory Medicine. Clin Chem. 2016;62:930-46

77. Kearney PM, Blackwell L, Collins R, Keech A, Simes J, Peto R, et al., Cholesterol Treatment Trialists' Collaboration. Efficacy of cholesterol lowering therapy in 18,686 people with diabetes in 14 randomised trials of statins: a meta-analysis. Lancet. 2008;371:117-25.

78. Van der Leeuw J, van Dieren S, Beulens JWJ, Boeing H, Spijkerman AMW, van der Graaf Y, et al. The validation of cardiovascular risk scores for patients with type 2 diabetes mellitus. Heart. 2015:101:222-9.

79. Gaede P, Lund-Andersen H, Parvins HH, Pedersen O. Effect of a multifactorial intervention on mortality in type 2 diabetes. N Engl J Med. 2008;358:580-91

80. Inzucchi SE, Bergenstal RM, Buse JB, Diamant M, Ferrannini E, Nauck M, et al Management of hyperglycemia in type 2 diabetes, 2015: a patient-centered approach: update to a position statement of the American Diabetes Association and the European Association for the Study of Diabetes. Diabetes Care. 2015;38:140-9.

81. Zinman B, Wanner C, Lachin JM, Fitchett D, Bluhmki E, Hantel S, et al. Empagliflozin, cardiovascular outcomes, and mortality in type 2 diabetes. N Engl J Med. 2015;373:2117-28.

82. Marso SP, Daniels GH, Brown-Frandsen K, Kristensen P, Mann JF, Nauck MA, et al. Liraglutide and cardiovascular outcomes in type 2 diabetes. N Engl J Med. 2016;375:311-22.

83. Emdin CA, Rahimi K, Neal B, Callender T, Perkovic V, Patel A. Blood pressure lowering in type 2 diabetes: a systematic review and meta-analysis. JAMA. 2015;313:603-15.

84. The SPRINT research Group. A randomized trial of intensive versus standard blood-pressure control. N Engl J Med. 2015;373:2103-16.

85. Williamson JD, Supiano MA, Applegate WB, Berlowitz DR, Campbell RC, Chertow GM, et al., for the SPRINT Research Group. Intensive vs standard blood pressure control and cardiovascular disease outcomes in adults aged $>75$ years. A randomized clinical trial. JAMA. 2016;315:2673-82.

86. Ettehad D, Emdin CA, Kiran A, Callender T, Emberson J, Chalmers J, et al. Blood pressure lowering for prevention of cardiovascular disease and death: a systematic review and meta-analysis. Lancet. 2016;387:957-67.

87. Lonn EM, Bosch J, López-Jaramillo P, Zhu J, Liu L, Pais P, et al., for the HOPE-3 Investigators. Blood-pressure lowering in intermediate-risk persons without cardiovascular disease. N Engl J Med. 2016;374:2009-20.

88. Yusuf S, Lonn E, Pais P, Bosch J, López-jaramillo P, Zhu J, et al., for the HOPE3 Investigators. Blood-Pressure and cholesterol lowering in persons without cardiovascular disease. N Engl J Med. 2016;374:2032-43.

89. Guirguis-Blake JM, Evans CV, Senger CA, Rowland MG, O'Connor EA, Whitlock EP. Aspirin for the Primary Prevention of Cardiovascular Events: A Systematic Evidence Review for the U.S. Preventive Services Task Force. Rockville MD; 2015.

90. A randomised, blinded, trial of clopidogrel versus aspirin in patients at risk of ischaemic events (CAPRIE). CAPRIE Steering Committee. Lancet. 1996;348:1329-39.

91. National Institute for Health and Care Excellence. Prevention of cardiovascular disease at the population level. London: National Institute for Health and Care Excellence, 2010.

92. Bureau Européen des Unions de Consommateurs. The consumer case for EU legal restrictions on the use of artificial trans-fats in food. Position paper. Brussels: European Union, 2014. Disponible en: http://www.beuc.eu/publications/ beuc-x-2014-010_the_consumer_case_for_eu_legal_restrictions_on_the_use_of artificial_trans.pdf
93. EU platform on diet, physical activity and health. European Union, 2005. Disponible en: http://ec.europa.eu/health/archive/ph_determinants life_style/nutrition/platform/docs/platform_charter.pdf

94. Gortmaker SL, Swinburn BA, Levy D, Carter R, Mabry PL, Finegood DT, et al. Changing the future of obesity: science, policy, and action. Lancet. 2011;378:838-47.

95. Royo-Bordonada MÁ, León-Flández K, Damián J, Bosqued-Estefanía MJ, MoyaGeromini MÁ, López-Jurado L. The extent and nature of food advertising to children on Spanish television in 2012 using an international foodbased coding system and the UK nutrient profiling model. Public Health. 2016;137:88-94

96. Royo-Bordonada MA, Bosqued-Estefanía MJ, Damián J, López-Jurado L, MoyaGeromini MA. Nutrition and health claims in products directed at children via television in Spain in 2012. Gac Sanit. 2016;30:221-6.

97. Prieto-Castillo L, Royo-Bordonada MA, Moya-Geromini A. Information search behaviour, understanding and use of nutrition labeling by residents of Madrid, Spain. Public Health. 2015;129:226-36.

98. Hawley KL, Roberto CA, Bragg MA, Liu PJ, Schwartz MB, Brownell KD. The science on front-of-package food labels. Public Health Nutr. 2013;16: 430e9.

99. Powell LM, Chriqui JF, Khan T, Wada R, Chaloupka FJ. Assessing the potential effectiveness of food and beverage taxes and subsidies for improving public health: a systematic review of prices, demand and body weight outcomes. Obesity Rev. 2013;14:110-28.

100. Hawkes C, Smith TG, Jewell J, Wardle J, Hammond RA, Friel S, et al. Smart food policies for obesity prevention. Lancet. 2015;385:2410-21.

101. Colchero MA, Popkin BM, Rivera JA, Ng SW. Beverage purchases from stores in Mexico under the excise tax on sugar sweetened beverages: observational study. BMJ. 2016;352:6704.

102. Ministerio de Sanidad, Servicios Sociales e Igualdad. Encuesta Nacional de Salud de España 2006. Disponible en: http://www.msssi.gob.es/estadEstudios estadisticas/encuestaNacional/encuesta2006.htm

103. Ministerio de Sanidad, Servicios Sociales e Igualdad. Encuesta Nacional de Salud de España 2011/12. Disponible en: http://www.msssi.gob.es/estadEstudios estadisticas/encuestaNacional/encuesta2011.htm

104. Ortún V, López-Valcárcel 2 G, Pinilla J. Tax on sugar sweetened beverages in Spain. Rev Esp Salud Publica. 2016;90:e1-13.

105. Geaney F, Kelly C, Greiner BA, Harrington JM, Perry IJ, Beirne P. The effectiveness of workplace dietary modification interventions: a systematic review. Prev Med. 2013;57:438-47.

106. Mayne SL, Auchincloss AH, Michael YL. Impact of policy and built environment changes on obesity-related outcomes: A systematic review of naturally occurring experiments. Obes Rev. 2015;16:362-75.

107. Documento de consenso sobre la alimentación en los centros educativos. Madrid: Consejo Interterritorial del Sistema Nacional de Salud 2010. Disponible en: http://www.naos.aesan.msssi.gob.es/naos/ficheros/ escolar/DOCUMENTO DE-CONSENSO PARA WEB.pdf

108. Ley $17 / 2011$, de 5 de julio, de Seguridad alimentaria y nutrición. Boletín Oficial del Estado BOE. 2011. 160: 71283-319. Disponible en: https://www.boe.es diario_boe/txt.php?id=BOE-A-2011-11604

109. Monroy-Parada DX, Moya MA, Bosqued MJ, López L, Rodríguez-Artalejo F Royo-Bordonada MÁ. Vending machines of food and beverages and nutritional profile of their products at schools in Madrid, Spain, 2014-2015. Rev Esp Salud Publica. 2016;90:e1-9.

110. Global Advocacy Council for Physical Activity, International Society for Physical Activity and Health. The Toronto Charter for Physical Activity: a global call for action. J Phys Act Health. 2010;7 Suppl.3:S370-85.

111. Colcombe SJ, Kramer AF, Erickson KI, Scalf P, McAuley E, Cohen NJ, et al. Cardiovascular fitness, cortical plasticity, and aging. Proc Natl Acad Sci USA 2004;101:3316-21.

112. Leyk D, Rohde U, Hartmann ND, Preuss PA, Sievert A, Witzki A. Results of a workplace health campaign: what can be achieved? Dtsch Arztebl Int 2014;111:320-7.

113. Mozaffarian D, Afshin A, Benowitz NL, Bittner V, Daniels SR, Franch HA, et al. Population approaches to improve diet, physical activity, and smoking habits: a scientific statement from the American Heart Association. Circulation. 2012:126:1514-63.

114. Jorgensen T, Capewell S, Prescott E, Allender S, Sans S, Zdrojewski T, et al Population-level changes to promote cardiovascular health. Eur J Prev Cardiol. 2013:20:409-21

115. Brien SE, Ronksley PE, Turner BJ, Mukamal KJ, Ghali WA. Effect of alcoho consumption on biological markers associated with risk of coronary heart disease: systematic review and meta-analysis of interventional studies. BM]. 2011;342:d636.

116. Mukamal KJ, Chen CM, Rao SR, Breslow RA. Alcohol consumption and cardiovascular mortality among U.S. adults, 1987 to 2002. J Am Coll Cardiol 2010;55:1328-35

117. Newby DE, Mannucci PM, Tell GS, Baccarelli AA, Brook RD, Donaldson K, et al. Expert position paper on air pollution and cardiovascular disease. Eur Heart J 2015;36:83-93.

118. Allen JK, Dennison-Himmelfarb CR, Szanton SL, Bone L, Hill MN, Levine DM, et al. Community Outreach and Cardiovascular Health $(\mathrm{COACH})$ Trial: a randomized, controlled trial of nurse practitioner/community health worker cardiovascular disease risk reduction in urban community health centers. Circ Cardiovasc Qual Outcomes. 2011:4:595-602. 
119. Anderson L, Taylor RS, Cardiac rehabilitation for people with heart disease: an overview of Cochrane systematic reviews. Cochrane Database Syst Rev. 2014;12:CD011273.

120. Jorstad HT, von Birgelen C, Alings AM, Liem A, van Dantzig JM, Jaarsma W, et al. Effect of a nurse-coordinated prevention programme on cardiovascular risk after an acute coronary syndrome: main results of the RESPONSE randomised trial. Heart. 2013;99:1421-30.

121. Ministerio de Sanidad, Política Social e Igualdad. Estrategia en Cardiopatía Isquémica del Sistema Nacional de Salud. Madrid: Ministerio de Sanidad, Política Social e Igualdad; 2011. Disponible en: http://www.mspsi.gob.es/ organizacion/sns/planCalidadSNS/docs/cardiopatia_isquemica/Estrategia Cardiopatia_Isquemica.pdf

122. Ministerio de Sanidad y Política Social. Estrategia en Ictus del Sistema Nacional de Salud. Madrid: Ministerio de Sanidad y Política Social; 2009. Disponible en:
http://www.msc.es/organizacion/sns/planCalidadSNS/docs/EstrategiaIctus SNS.pdf

123. Ministerio de Sanidad, Servicios Sociales e Igualdad. Estrategia en Diabetes del Sistema Nacional de Salud. Actualización. Madrid: Ministerio de Sanidad, Servicios Sociales e Igualdad; 2012. Disponible en: http://www.msps.es/ organizacion/sns/planCalidadSNS/pdf/excelencia/cuidadospaliativosdiabetes/DIABETES/Estrategia_en_diabetes_del_SNS_Accesible.pdf

124. Agencia Española de Seguridad Alimentaria. Estrategia para la Nutrición, Actividad Física y Prevención de la Obesidad. Madrid: 2005. Disponible en: http://www.aecosan.msssi.gob.es/AECOSAN/docs/documentos/nutricion/ estrategianaos.pdf

125. Royo-Bordonada MA, Lobos JM, Brotons C, Villar F, de Pablo C, Armario P, et al. El estado de la prevención cardiovascular en España. Med Clin (Barc). 2014;142:7-14. 